\title{
THE STABILITY OF MATTER: FROM ATOMS TO STARS
}

\author{
ELLIOTT H. LIEB
}

Why is ordinary matter (e.g., atoms, molecules, people, planets, stars) as stable as it is? Why is it the case, if an atom is thought to be a miniature solar system, that bringing very large numbers of atoms together (say $10^{30}$ ) does not produce a violent explosion? Sometimes explosions do occur, as when stars collapse to form supernovae, but normally matter is well behaved. In short, what is the peculiar mechanics of the elementary particles (electrons and nuclei) that constitute ordinary matter so that the material world can have both rich variety and stability?

The law of motion that governs these particles is the quantum (or wave) mechanics discovered by Schrödinger [SE] in 1926 (with precursors by Bohr, Heisenberg, Sommerfeld and others). Everything we can sense in the material world is governed by this theory and some of its consequences are quite dramatic, e.g., lasers, transistors, computer chips, DNA. (DNA may not appear to be very quantum mechanical, but notice that it consists of a very long, thin, complex structure whose overall length scale is huge compared to the only available characteristic length, namely the size of an atom, and yet it is stable.) But we also see the effects of quantum mechanics, without realizing it, in such mundane facts about stability as that a stone is solid and has a volume which is proportional to its mass, and that bringing two stones together produces nothing more exciting than a bigger stone.

The mathematical proof that quantum mechanics gives rise to the observed stability is not easy because of the strong electric forces among the elementary constituents (electrons and nuclei) of matter. The big breakthrough came in the mid sixties when Dyson and Lenard [DL] showed, by a complicated proof, that stability

Received by the editors August 15, 1989.

1980 Mathematics Subject Classification (1985 Revision). Primary 81H99, $81 \mathrm{M} 05,85 \mathrm{~A} 15$; Secondary 81C99, 82A15.

This paper was presented as the Sixty-Second Josiah Willard Gibbs Lecture on January 11, 1989 at the 95th annual meeting of the American Mathematical Society in Phoenix, Arizona.

Work partially supported by U.S. National Science Foundation grant PHY-8515288-A03. 
is, indeed, a consequence of quantum mechanics. (Part of their motivation came from earlier work by Van Hove, Lee and Yang, van Kampen, Wils, Mazur, van der Linden, Griffiths, Dobrushin, and especially Fisher and Ruelle who formulated the problem and showed how to handle certain well chosen, but unrealistic forces.) This was a milestone but there was room left for improvement since their results had certain drawbacks and did not cover all possible cases; for instance, it turns out that quantum mechanics, which was originally conceived to understand atoms, is also crucial for understanding why stars do not collapse. Another problem was that they proved what is called here stability of the second kind while the existence of the thermodynamic limit (Theorem 3 below), which is also essential for stability, required further work [LL]. The full story has now, two decades later, mostly been sorted out, and that is the subject of this lecture. The answer contains a few surprises, some of which are not even discussed in today's physics textbooks.

No physics background will be assumed of the reader, so Part I reviews some basic facts. Part II contains a synopsis of the aspects of quantum mechanics needed here. Part III treats the simplest system - the hydrogen atom, and Part IV introduces the strange Pauli exclusion principle for many electrons and extends the discussion to large atoms. Part V deals with the basic issue of the stability of matter (without relativistic effects) while Part VI treats hypothetical, but interesting, matter composed of bosons. Part VII treats the problems introduced by the special theory of relativity. Finally, Part VIII applies the results of Part VII to the structure of stars.

\section{PART I. THE PHYSICAL FACTS AND THEIR PREQUANTUM INTERPRETATION}

While it is certainly possible to present the whole story in a purely mathematical setting, it is helpful to begin with a brief discussion of the physical situation.

The first elementary constituent of matter to be discovered was the electron (J.J. Thomson, 1897). This particle has a negative electric charge (denoted by $-e$ ) and a mass, $m$. It is easy to produce a beam of electrons (e.g., in a television tube) and use it to measure the ratio $e / m$ quite accurately. The measurement of $e$ alone is much trickier (Millikan 1913). The electron can be considered to be a point, i.e., it has no presently discernible geometric structure. Since matter is normally electrically neutral (otherwise we would feel electric fields everywhere), there must also be another 
constituent with positive charge. One of the early ideas about this positive object was that it is a positively charged ball of a radius about equal to the radius of an atom, which is approximately $10^{-8}$ $\mathrm{cm}$. (This atomic radius is known, e.g., by dividing the volume of a solid, which is the most highly compressed form of matter, by the number of atoms in the solid.) The electrons were thought to be stuck in this charged ball like raisins in a cake; such a structure would have the virtue of being quite stable, almost by fiat. This nice picture was destroyed, however, by Rutherford's classic 1903 experiment which showed that the positive entities were also essentially points. (He did this by scattering positively charged helium nuclei through thin metal foils and by showing that the distribution of scattering angles was the same as for the Kepler problem in which the trajectories are hyperbolas; in other words, the scatterers were effectively points-not extended objects.)

The picture that finally emerged was the following. Ordinary matter is composed of two kinds of particles: the point electrons and positively charged nuclei. There are many kinds of nuclei, each of which is composed of positively charged protons and chargeless neutrons. While each nucleus has a positive radius, this radius (about $10^{-13} \mathrm{~cm}$ ) is so small compared to any length we shall be considering that it can be taken to be zero for our purposes. The simplest nucleus is the single proton (the nucleus of hydrogen) and it has charge $+e$. The number of protons in a nucleus is denoted by $z$ and the values $z=1,2, \ldots, 92$, except for $z=43,61,85$, are found in nature. Some of these nuclei, e.g., all $84 \leq z \leq 92$, are unstable (i.e., they eventually break apart spontaneously) and we see this instability as naturally occurring radioactivity, e.g., radium. Nuclei with the missing $z$ values 43 , 61,85 , as well as those with $92<z \leq 109$ have all been produced artificially, but they decay more or less quickly [AM]. Thus, the charge of a naturally occurring nucleus can be $+e$ up to $+92 e$ (except for 43, 61, 85), but, as mathematicians often do, it is interesting to ask questions about "the asymptotics as $z \rightarrow \infty$ " of some problems. Moreover, in almost all cases we shall consider here, the physical constraint that $z$ is an integer need not and will not be imposed. The other constituent-the neutrons-will be of no importance to us until we come to stars. They merely add to the mass of the nucleus, for they are electrically neutral. For each given $z$ several possible neutron numbers actually occur in nature; these different nuclei with a common $z$ are called isotopes of each other. For example, when $z=1$ we have the hydrogen nucleus ( 1 proton) and the deuterium nucleus (1 proton and 1 neutron) 
which occur naturally, and the tritium nucleus $(1$ proton and 2 neutrons) which is artificial and decays spontaneously in about 12 years into a helium nucleus and an electron, but which is important for hydrogen bombs. Isolated neutrons are also not seen naturally, for they decay in about 13 minutes into a proton and an electron.

Finally, the nuclear mass, $M$, has to be mentioned. It satisfies $z M_{p} \leq M \leq 3 z M_{p}$ where $M_{p}=1837 \mathrm{~m}$ is the mass of a proton. Since the nuclear mass is huge compared to the electron mass, $m$, it can be considered to be infinite for most purposes, i.e., the nuclei can be regarded as fixed points in $\mathbf{R}^{3}$, although the location of these points will eventually be determined by the requirement that the total energy of the electron-nucleus system is minimized. A similar approximation is usually made when one considers the solar system; to calculate the motion of the planets the sun can be regarded as fixed.

The forces between these constituents of matter (electrons and nuclei) is given by Coulomb's inverse square law of electrostatics: If two particles have charges $q_{1}$ and $q_{2}$ and locations $x_{1}$ and $x_{2}$ in $\mathbf{R}^{3}$ then $F_{1}$-the force on the first due to the second-is minus $F_{2}$-the force on the second due to the first-and is given by

$$
-F_{2}=F_{1}=q_{1} q_{2} \frac{\left(x_{1}-x_{2}\right)}{\left|x_{1}-x_{2}\right|^{3}} .
$$

(Later on, when stars are discussed, the gravitational force will have to be introduced.) If $q_{1} q_{2}<0$ then the force is attractive; otherwise it is repulsive. This force can also be written as minus the gradient (denoted by $\nabla$ ) of a potential energy function

$$
W\left(x_{1}, x_{2}\right)=q_{1} q_{2} \frac{1}{\left|x_{1}-x_{2}\right|},
$$

that is

$$
F_{1}=-\nabla_{1} W \text { and } F_{2}=-\nabla_{2} W
$$

If there are $N$ electrons located at $\underline{X}=\left(x_{1}, \ldots, x_{N}\right)$ with $x_{i} \in \mathbf{R}^{3}$, and $k$ nuclei with positive charges $\underline{Z}=\left(z_{1}, \ldots, z_{k}\right)$ and located at $\underline{R}=\left(R_{1}, \ldots, R_{k}\right)$ with $R_{i} \in \mathbf{R}^{3}$, the total-potential energy function is then

$$
W(\underline{X})=-A(\underline{X})+B(\underline{X})+U
$$


with

$$
\begin{aligned}
A(\underline{X}) & =e^{2} \sum_{i=1}^{N} V\left(x_{i}\right) \\
V(x) & =\sum_{j=1}^{k} z_{j}\left|x-R_{j}\right|^{-1} \\
B(\underline{X}) & =e^{2} \sum_{1 \leq i<j \leq N}\left|x_{i}-x_{j}\right|^{-1} \\
U & =e^{2} \sum_{1 \leq i<j \leq k} z_{i} z_{j}\left|R_{i}-R_{j}\right|^{-1} .
\end{aligned}
$$

The $A$ term is the electron-nucleus attractive potential energy, with $e V(x)$ being the electric potential of the nuclei. $B$ is the electron-electron repulsive energy and $U$ is the repulsive energy of the nuclei. $A, B, U$ and $V$ depend on $\underline{R}$ and $\underline{Z}$, which are fixed and therefore do not appear explicitly in the notation. It is then the case that the force on the $i$ th particle is

$$
F_{i}=-\nabla_{i} W
$$

In the case of an atom, $k=1$ by definition. The case $k>1$ will be called the molecular case, but it includes not only the molecules of the chemist but also solids, which are really only huge molecules.

So far this is just classical electrostatics and we turn next to classical dynamics. Newton's law of motion is (with a dot denoting $\frac{d}{d t}$, where $t$ is the time)

$$
m \ddot{x}_{i}=F_{i}
$$

This law of motion, which is a system of second order differential equations, is equivalent to the following system of first order equations. Introduce the Hamiltonian function which is the function on the phase space $\mathbf{R}^{6 N}=\left(\mathbf{R}^{3} \times \mathbf{R}^{3}\right)^{N}$ given by

$$
H(\underline{P}, \underline{X})=\frac{1}{2 m} \sum_{i=1}^{N} p_{i}^{2}+W(\underline{X}) .
$$

The notation $\underline{P}=\left(p_{1}, \ldots, p_{N}\right)$ with $p_{i}$ in $\mathbf{R}^{3}$ is used, and the quantity

$$
T=\frac{1}{2 m} \sum_{i=1}^{N} p_{i}^{2}
$$


is called the kinetic energy. The equations of motion (1.10) are equivalent to the following first order system in $\mathbf{R}^{6 N}$

$$
\begin{aligned}
v_{i} \equiv \dot{x}_{i} & =\frac{\partial H}{\partial p_{i}} \\
\dot{p}_{i} & =-\frac{\partial H}{\partial x_{i}} .
\end{aligned}
$$

The velocity of the $i$ th electron is $v_{i}$ and $p_{i}$ is called its momentum: $p_{i}=m v_{i}$ by the first equation in (1.13).

From (1.13) it will be seen that $H(\underline{P}, \underline{X})$ is constant throughout the motion, i.e., $d H(\underline{P}(t), \underline{X}(t)) / d t=0$. This fixed number is called the energy and is denoted by $E$; it depends, of course, on the trajectory, and it is important to note that it can take all values in $(-\infty, \infty)$.

Another interesting fact about the flow defined by (1.13), but one which will not be important for us, is that it preserves Lebesgue measure $d x_{1} \cdots d x_{N} d p_{1} \cdots d p_{N}$ on $\mathbf{R}^{6 N}$; this is Liouville's theorem and it follows from the fact that the vector field that defines the flow, $\left(\partial H / \partial p_{1}, \ldots, \partial H / \partial p_{N},-\partial H / \partial x_{1}, \ldots,-\partial H / \partial x_{N}\right)$, is divergence free. This theorem is one important reason for introducing the Hamiltonian formalism, for it permits a geometric interpretation of classical mechanics and is crucial for ergodic theory and statistical mechanics. The analogue in quantum mechanics turns out to be that quantum mechanical time evolution is given by a one parameter unitary group in Hilbert space (see (2.18)) but time evolution will not concern us here.

Consider the simplest possible case, neutral hydrogen, with $z=$ 1 (a proton) and one electron $(N=1$ and $k=1)$. With the proton fixed at the origin (i.e., $R_{1}=0$ ) the Hamiltonian is $p^{2} / 2 m-Z e^{2}|x|^{-1}$ and classical bound orbits (i.e., orbits which do not escape to infinity) of the electron are well known to be the ellipses of Kepler with the origin as a focus. These can pass as close as we please to the proton. Indeed, in the degenerate case the orbit is a radial line segment and in such an orbit the electron passes through the nucleus. One measure of average closeness of the electron to the nucleus in an orbit is the energy $E$, which is always negative for a bound orbit. Moreover $E$ can be arbitrarily negative because the electron can be arbitrarily close to the nucleus and also have arbitrarily small kinetic energy $T$. A consequence of this fact is that the hydrogen atom would be physically unstable; in a gas of many atoms another particle or atom could collide with our atom and absorb energy from it. After many such collisions our electron could find itself in a tiny orbit around the nucleus 
and our atom would no longer be recognizable as an object whose radius is supposed to be $10^{-8} \mathrm{~cm}$. Each atom would be an infinite source of energy which could be transmitted to other atoms or to radiation of electromagnetic waves.

The problem was nicely summarized by Jeans [J] in his 1915 textbook.

"There would be a very real difficulty in supposing that the (force) law $1 / r^{2}$ held down to zero values of $r$. For the force between two charges at zero distance would be infinite; we should have charges of opposite sign continually rushing together and, when once together, no force would be adequate to separate them... Thus the matter in the universe would tend to shrink into nothing or to diminish indefinitely in size."

The inability to account for stable atoms in terms of classical trajectories of pointlike charged particles was the major problem of prequantum physics. Since the existence of atoms and molecules was largely inferential in those days (nowadays we can actually "see" atoms with the tunneling electron microscope), the inability to account for their structure even led some serious people to question their existence-or at least to question the nice pictures drawn by chemists. The main contribution of quantum mechanics was to provide a quantitative theory that "explains" why the electron cannot fall into the nucleus. In brief, when the electron is close to the nucleus its kinetic energy - which could be zero classically-is forced to increase in such a way that the total energy (1.11) goes to $+\infty$ as the average distance $|x|$ goes to zero. This property is known as the uncertainty principle.

\section{PART II. QUANTUM MECHANICS IN A NUTSHELL}

Schrödinger's answer to the problem of classical mechanics was the following. While an electron is truly a point particle, its state at any given time cannot be described by a point $x \in \mathbf{R}^{3}$ and a momentum $p \in \mathbf{R}^{3}$ (or velocity $v=\frac{1}{m} p$ ) as in the classical view. Instead the state of an electron is a (complex valued) function $\psi$ in $L^{2}\left(\mathbf{R}^{3}\right)$. Any $\psi$ will do provided it satisfies the normalization condition

$$
\|\psi\|_{2}^{2}=\int_{\mathbf{R}^{3}}|\psi(x)|^{2} d x=1 .
$$

(Actually, this statement is not accurate; an electron has a property called spin, and the mathematical expression of this fact is 
that $\psi$ is really in $L^{2}\left(\mathbf{R}^{3} ; \mathbf{C}^{2}\right)$, i.e., $\psi(x)$ is a two-component spinor, $\psi(x)=\left(\psi_{1}(x), \psi_{2}(x)\right)$ with each $\psi_{i} \in L^{2}\left(\mathbf{R}^{3}\right)$. This complication-which does not affect the present discussion very much-will frequently be ignored here.) Thus, the state of an electron is a point $\psi$ in an infinite dimensional Hilbert space instead of a point $(p, x)$ in $\mathbf{R}^{6}$.

The interpretation of $\psi$, due to M. Born [BM] (Jammer's book [JM] can be consulted for historical details) is that

$$
\rho_{\psi}(x) \equiv|\psi(x)|^{2}
$$

is the probability density for finding the electron at $x$. The expected value of the potential energy $W(x)$ in the state $\psi$ of one electron is then

$$
W_{\psi}=\int_{\mathbf{R}^{3}} \rho_{\psi}(x) W(x) d x .
$$

To localize an electron at a point $x_{0}$ would require $|\psi|^{2}=\delta_{x_{0}}$, where $\delta_{x_{0}}$ is Dirac's delta measure, but this is obviously not the square of an $L^{2}\left(\mathbf{R}^{3}\right)$ function. Thus, it no longer makes sense to speak of an electron unambiguously located at a single point $x_{0}$.

What about the kinetic energy $T=p^{2} / 2 m$ ? To define $T$ we first consider the Fourier transform of $\psi$ scaled by the number $h$ and defined by

$$
\widehat{\psi}(p)=h^{-3 / 2} \int_{\mathbf{R}^{3}} \psi(x) \exp \left[-\frac{2 \pi i}{h}(p, x)\right] d x .
$$

Usually $h$ is taken to be 1 or $2 \pi$ in textbooks but any $h$ can be used. Although $\widehat{\psi}$ depends on the choice of $h$, Plancherel's formula is always the following:

$$
\int_{\mathbf{R}^{3}}|\widehat{\psi}(p)|^{2} d p=1
$$

The constant $h$ relevant for quantum mechanics is Planck's constant; it is not arbitrary and has to be determined experimentally in order to satisfy the following physical interpretation of $|\widehat{\psi}(p)|^{2}$. In view of $(2.5),|\widehat{\psi}(p)|^{2}$ can be regarded as a probability density - it is the probability density of the electron having a momentum $p$. With this interpretation, the kinetic energy of the electron in the state $\psi$ is

$$
T_{\psi}=\frac{1}{2 m} \int_{\mathbf{R}^{3}}|\widehat{\psi}(p)|^{2} p^{2} d p=\frac{1}{2 m} \hbar^{2} \int_{\mathbf{R}^{3}}|\nabla \psi(x)|^{2} d x,
$$


where, in a customary notation, $\hbar=h / 2 \pi$. The role of $h$ in the definition (2.4) is brought into focus by the right side of (2.6). Numerically $\hbar=1.05 \times 10^{-27}$ erg seconds.

Another way to formulate (2.3) to (2.6) is to think of $L^{2}\left(\mathbf{R}^{3}\right)$ as a Hilbert space with inner product $(\psi, \phi)=\int \bar{\psi}(x) \phi(x) d x$ so that

$$
W_{\psi}=(\psi, W \psi)
$$

The momentum $p$ is replaced by the self adjoint operator

$$
p=-i \hbar \nabla \text { and } p^{2}=-\hbar^{2} \Delta
$$

with $\Delta$ being the Laplacian $\partial^{2} / \partial x^{2}+\partial^{2} / \partial y^{2}+\partial^{2} / \partial z^{2}$. Then the kinetic energy is (after integrating by parts)

$$
2 m T_{\psi}=(p \psi, p \psi)=\hbar^{2}\|\nabla \psi\|_{2}^{2}=\left(\psi, p^{2} \psi\right)
$$

These concepts can be generalized to the many-body case of $N$ electrons by replacing $\mathbf{R}^{3}$ by $\mathbf{R}^{3 N}$. Then $\psi(x)$ is replaced by $\psi(\underline{X})=\psi\left(x_{1}, \ldots, x_{N}\right)$ in $\bigotimes_{1}^{N} L^{2}\left(\mathbf{R}^{3}\right) \approx L^{2}\left(\mathbf{R}^{3 N}\right)$. (If spin is included then we use $\otimes_{1}^{N} L^{2}\left(\mathbf{R}^{3}, \mathbf{C}^{2}\right) \approx L^{2}\left(\mathbf{R}^{3 N} ; \mathbf{C}^{2^{N}}\right)$.) A different generalization that might leap to the reader's mind would be to replace $\psi(x)$ by an $N$-tuple of functions on $\mathbf{R}^{3}$; that would lead to a nonlinear theory and would be wrong. The normalization condition is

$$
\int_{\mathbf{R}^{3 N}}|\psi(\underline{X})| d x_{1} \cdots d x_{N}=1
$$

and $|\psi(\underline{X})|^{2}$ is the joint probability density for finding the electrons at $x_{1}, \ldots, x_{N}$. The potential and kinetic energies are given by

$$
\begin{aligned}
W_{\psi} & =\int_{\mathbf{R}^{3 N}} W(\underline{X})|\psi(\underline{X})|^{2} d x_{1} \cdots d x_{N} \\
T_{\psi} & =\frac{1}{2 m} \hbar^{2} \sum_{i=1}^{N} \int_{\mathbf{R}^{3 N}}\left|\left(\nabla_{x_{i}} \psi\right)(\underline{X})\right|^{2} d x_{1} \cdots d x_{N} .
\end{aligned}
$$

The energy of $\psi$ is then

$$
E_{\psi}=T_{\psi}+W_{\psi} .
$$

The problem that will concern us here is to find a lower bound to the ground state energy

$$
E=E(N, k, \underline{Z}, \underline{R})=\inf _{\psi} E_{\psi},
$$


because the boundedness of $E$ precludes the kind of collapse that Jeans was worried about. (Recall that $E_{\psi}$ depends on $N, k, \underline{Z}$ and $\underline{R}$ since $W(\underline{X})$ does.) In (2.13) $\psi$ is required to satisfy the normalization condition (2.9). It will also be required to satisfy another condition-the Pauli exclusion principle - which will be explained later.

The absolute ground state energy is

$$
E(N, k, \underline{Z})=\inf _{\underline{R}} E(N, k, \underline{Z}, \underline{R})
$$

and it is the ground state energy when the nuclei are placed in the most favorable locations.

There are two notions of stability

(A) STABILITY OF THE FIRST KIND. $E(N, k, \underline{Z}, \underline{R})$ is finite for every $N, k, \underline{Z}$ and $\underline{R}$.

(B) STABILITY OF THE SECOND KIND. There is a nonnegative function $z \mapsto A(z)$ such that for all $N$ and $k$

$$
E(N, k, \underline{Z}) \geq-A(z)(N+k)
$$

provided each $z_{j} \leq z$. Recall that $z_{j} \leq 92$ in nature. The significance of the linear law (2.15) will be discussed later in Part V.

The infimum in (2.13) may or may not be attained by some $\psi$. If it is, a minimizing $\psi$, which may not be unique, is called a ground state. It satisfies the linear Euler-Lagrange equation (also known as the time independent Schrödinger equation)

$$
H \psi=E \psi
$$

where $H$ is the Schrödinger Hamiltonian, namely the selfadjoint second order elliptic differential operator

$$
H=-\frac{1}{2 m} \hbar^{2} \sum_{i=1}^{N} \Delta_{x_{i}}+W(\underline{X}) .
$$

In the previous Hilbert space notation, $E_{\psi}=(\psi, H \psi)$.

Quantum mechanics is the study of the eigenvalues of $H$, i.e., solutions of $H \psi=\lambda \psi$ with $\psi$ in $L^{2}$, and also the associated (time dependent) Schrödinger equation of the time evolution

$$
H \psi=-i \hbar \frac{\partial \psi}{\partial t}
$$

which is important in several contexts, especially in the subject of scattering theory. The eigenvalues $\lambda$ are called energy levels and differences between any two, $\lambda_{i}-\lambda_{j}$, is the energy carried away or absorbed by a photon when the system makes a "quantum jump" 
from eigenstate $\psi_{1}$ to eigenstate $\psi_{2}$. The fact that the system "jumps" from one eigenstate to another is a mysterious axiom of quantum mechanics (more precisely, quantum measurement theory) that need not concern us here, but it has the following important physical implication. According to Einstein, the frequency $\nu$ of a photon of energy $e$ is always $\nu=e / h$. Thus, by energy conservation the photon emitted or absorbed has frequency $\nu=\left(\lambda_{i}-\lambda_{j}\right) / h$. Since the $\lambda_{i}$ 's are discrete, the frequencies (or spectral lines) are also discrete. With classical mechanics we would expect to find all frequencies, and the experimental existence of discrete spectral lines is regarded in many textbooks as being the crucial problem that classical physics could not explain. However, Jeans's question about the existence of stable atoms capable of emitting these discrete spectral lines can evidently be regarded as a question of higher priority.

In this lecture we shall be interested only in the lowest eigenvalue of $H$, which is $E$. Equation (2.18) is therefore irrelevant here. Also (2.16) will not be invoked because we shall deal directly with the minimization problem (2.13) which, incidentally, avoids the technically difficult question of the selfadjointness of $H$.

\section{PART III. THE HYDROGENIC ATOM AND SOBOLEV'S INEQUALITY}

In Parts III-VI we shall use units in which $\hbar^{2} / 2 m=h^{2} / 8 \pi^{2} m=$ 1 and $e=1$. This can always be done by changing the length scale $x \rightarrow\left(\hbar^{2} / 2 m e^{2}\right) x^{\prime}$. The original energy $E$ is related to the new energy $E^{\prime}$ (which will henceforth be denoted simply as $E$ ) by $E=\left(2 m e^{2} / \hbar^{2}\right) E^{\prime}$.

The problem for hydrogen is to minimize

$$
E_{\psi}=T_{\psi}+W_{\psi}=\int_{\mathbf{R}^{3}}|\nabla \psi(x)|^{2} d x-z \int_{\mathbf{R}^{3}}|\psi(x)|^{2}|x|^{-1} d x
$$

with $\int|\psi|^{2}=1$. We can take $\psi$ to be real, as is true in all the problems considered here, because the real and imaginary parts of $\psi$ appear independently in (3.1) and (2.1). Of course $z=1$ for hydrogen but it will be useful later to consider (3.1) with arbitrary $z>0$.

The fact that $T_{\psi}$ controls $W_{\psi}$, thereby making $E$ finite, is most clearly seen with the Sobolev inequality

$$
T_{\psi}=\|\nabla \psi\|_{2}^{2} \geq S\|\psi\|_{6}^{2}=S\left\{\int_{\mathbf{R}^{3}} \rho_{\psi}(x)^{3} d x\right\}^{1 / 3}=S\left\|\rho_{\psi}\right\|_{3}
$$


for any $\psi \in L^{2}\left(\mathbf{R}^{3}\right)$ (not necessarily normalized). Here $S=$ $3(\pi / 2)^{4 / 3} \approx 5.5$ and we recall $(2.2)$. The important point about (3.2) is that it bounds $T_{\psi}$ in terms of $\rho_{\psi}$; the derivatives have been eliminated. The ground state energy can be bounded from below using (3.2), and only the fact that $\int \rho_{\psi}=1$, by

$$
E \geq \inf _{\rho}\left\{S\|\rho\|_{3}-z \int_{\mathbf{R}^{3}}|x|^{-1} \rho(x) d x\right\}
$$

where the infimum is over all nonnegative $\rho$ with $\int \rho=1$. This minimization problem is an easy exercise and the answer is

$$
E \geq-\frac{1}{3} z^{2}
$$

This is a remarkably good bound since the correct answer, which is obtained by solving (2.16), is $E=-z^{2} / 4$.

Before moving on, it is important to understand heuristically why (3.1) leads to $E \approx-z^{2}$. If $\psi$ is some nice function with $\|\psi\|_{2}=1$ and whose width is about $L$ (for example, the Gaussian $\psi(x)=$ (const.) $\left.\exp \left[-x^{2} / L^{2}\right]\right)$, then $T_{\psi}$ is roughly $L^{-2}$. On the other hand, $W_{\psi}$ is roughly $-z L^{-1}$. Therefore, the problem of minimizing $E_{\psi}$ is roughly the same as

$$
E \approx \min _{L}\left\{\frac{1}{L^{2}}-\frac{z}{L}\right\}
$$

The minimizing $L$ is $L=2 / z$ and the minimum in (3.5) is $-z^{2} / 4$, which is the right answer. Indeed, the minimizing $\psi$ for $(3.1)$ is

$$
\phi(x)=(\text { const. }) e^{-z|x| / 2}
$$

which shows that the width of $\psi$ is, in fact, about $z^{-1}$. From this calculation, and recalling that $|\psi(x)|^{2}$ is the probability density for the electron, we learn that the size of a one-electron atom with nuclear charge $z$ decreases with $z$ like $z^{-1}$.

There is another version of (3.2) that is technically weaker, but more transparent and eventually more useful. Since $\int \rho_{\psi}=1$, a simple application of Hölder's inequality to the right side of (3.2) yields

$$
T_{\psi} \geq K^{1} \int_{\mathbf{R}^{3}} \rho_{\psi}(x)^{5 / 3} d x
$$

with $K^{1}=S$. The constant $K^{1}$ can be improved from $S$ to 9.57 , but that is conceptually unimportant. Instead of (3.3) we then 
have

$$
E \geq \inf _{\rho}\left\{\int_{\mathbf{R}^{3}}\left[K^{1} \rho(x)^{5 / 3}-|x|^{-1} \rho(x)\right] d x\right\}
$$

and this yields a result only slightly worse than (3.4), namely $-0.35 z^{2}$ with $K^{1}=9.57$. The important point is that (3.7) (with $K^{1}$ or with $S$ ) is just as good for our purposes as (3.3) because it yields the $z^{2}$ law, but the important advantage of (3.7) over (3.3) is that the integral comes with the exponent 1 in (3.7) instead of the exponent $1 / 3$ in (3.2). This gives (3.7) the form of the integral of an energy density $\rho_{\psi}(x)^{5 / 3}$. Independent "bumps" in $\rho_{\psi}$ give additive contributions to the right side of (3.7); in (3.2), by contrast, we have to take the cube root-which spoils the additivity.

The inequality (3.7) can be stated poetically as follows. An electron is like a rubber ball, or a fluid, with an energy density proportional to $\rho_{\psi}^{5 / 3}$. It costs energy to squeeze it and this accounts for the stability of atoms.

\section{PART IV. LARGE ATOMS AND THE PAULI EXCLUSION PRINCIPLE}

Suppose that $N=z$ and $z$ is large, say $z=50$. Since $N>1$, $W_{\psi}$ has two terms which come from $-A$ and $B$ in (1.4). The constant $U$ is zero since there is only one nucleus. The $B$ term is positive but it turns out to be small compared to the $A$ term and, for a preliminary orientation, can be neglected. We would then try to minimize

$$
\widetilde{E}_{\psi}=\sum_{i=1}^{N} \int_{\mathbf{R}^{3 N}}\left\{\left|\nabla_{x_{i}} \psi(\underline{X})\right|^{2}-z\left|x_{i}\right|^{-1} \mid\left(\left.\psi(\underline{X})\right|^{2}\right\} d x_{1} \cdots d x_{N}\right.
$$

The minimum with $\|\psi\|_{2}=1$ is then achieved by

$$
\psi(\underline{X})=\prod_{i=1}^{N} \phi\left(x_{i}\right),
$$

where $\phi(x)$ is the minimizer for (3.1) and is given in (3.6). This leads to

$$
\widetilde{E}=\inf _{\psi} \widetilde{E}_{\psi}=-N z^{2} / 4=-z^{3} / 4 .
$$

From this calculation we would be led to two conclusions: (1) The energy of an atom is finite (stability of the first kind) but it grows like $z^{3}$. (ii) Because the scale of $\phi$ is $z^{-1}$, the size of a 
large atom decreases with $z$ like $z^{-1}$. Conclusion (ii) is especially troublesome: large atoms are smaller than small atoms.

Both conclusions are false because of an additional axiom introduced by Pauli [P] in 1925 (and interpreted in the context of Schrödinger's wave mechanics by Dirac and Heisenberg in (1926)), namely the exclusion principle. This states that the allowed $\psi$ 's in the minimization problem (2.13) are the antisymmetric functions in $L^{2}\left(\mathbf{R}^{3 N}\right) \approx \otimes^{N} L^{2}\left(\mathbf{R}^{3}\right)$. (The reason for the word exclusion will be explained shortly.) In other words $\psi \in \Lambda^{N} L^{2}\left(\mathbf{R}^{3}\right)$, the antisymmetric tensor product of $L^{2}\left(\mathbf{R}^{3}\right)$. More explicitly

$$
\begin{aligned}
\psi\left(x_{1}, \ldots, x_{i}, \ldots, x_{j}, \ldots,\right. & \left.x_{N}\right)= \\
& -\psi\left(x_{1}, \ldots, x_{j}, \ldots, x_{i}, \ldots, x_{N}\right)
\end{aligned}
$$

for every $i \neq j$. If spin is taken into account then the condition is $\psi \in \bigwedge^{N} L^{2}\left(\mathbf{R}^{3} ; \mathbf{C}^{2}\right)$. (Equivalently, $\psi$ is an antisymmetric function in $L^{2}\left(\mathbf{R}^{3}, \mathbf{C}^{2^{N}}\right)$.) The physical significance of this peculiar antisymmetry restriction will not be discussed here, but it is related to the fact that electrons are indistinguishable.

A function in $\wedge^{N} L^{2}\left(\mathbf{R}^{3} ; \mathbf{C}^{2}\right)$ may be hard to think about-in which case the reader should ignore the complication of spin and think instead about $\bigwedge^{N} L^{2}\left(\mathbf{R}^{3}\right)$ without any essential loss. The following remarks may be useful, however. Elementary manipulations with the symmetric (or permutation) group $S_{N}$ show that our minimization problem (2.13) (or even the Schrödinger equation (2.16) or (2.18)) in $\bigwedge^{N} L^{2}\left(\mathbf{R}^{3} ; \mathbf{C}^{2}\right)$ is equivalent to solving the problem in a subset of the more familiar space $L^{2}\left(\mathbf{R}^{3 N}\right)$. This is the subset consisting all function $\psi=\psi\left(x_{1}, \ldots, x_{N}\right)$ with the property that there is some integer $1 \leq J \leq N$ such that $\psi$ is antisymmetric in the variables $x_{1}, \ldots, x_{J}$ and also antisymmetric in the variables $x_{J+1}, \ldots, x_{N}$ if $J<N$. No assertion is made about permuting variables $x_{i}$ and $x_{j}$ when $i \leq J<j$. Thus, it is as though there are two species of spinless particles ( $J$ of one kind and $N-J$ of the other kind), each of which satisfies the Pauli exclusion principle for spinless particles. The terminology that is employed to describe these "two kinds" of electrons is "electrons with spin up" and "electrons with spin down".

Particles such as electrons, protons and neutrons (and some, but not all nuclei) that satisfy the Pauli exclusion principle are called fermions. They always have spin. There is another kind of particle in nature-bosons. These have the restriction that $\psi$ is symmetric (there is only $L^{2}\left(\mathbf{R}^{3}\right)$ this time, not $L^{2}\left(\mathbf{R}^{3} ; \mathbf{C}^{2}\right)$ ). Every elementary particle is either a boson or a fermion. Fortunately, there are 
no negatively charged bosons which do not decay rapidly, for matter composed of negative bosons and positive bosons is not stable of the second kind-as will be seen later in Part VI.

It is a very general fact that the infimum in (2.13) over the full tensor product $\otimes^{N} L^{2}\left(\mathbf{R}^{3}\right)$ is the infimum over symmetric $\psi$ 's, i.e., bosons. To prove this fact, first replace $\psi$ (which is real and normalized) by $|\psi|$, which does not increase $E_{\psi}$ because $|\psi|^{2}$ and $|\nabla \psi|^{2}$ remain the same. Next, for any function $\Phi(\underline{X})$ we can construct a symmetric function $\Phi_{\sigma}(\underline{X})$ by summing over permutations, i.e.,

$$
\Phi_{\sigma}(\underline{X})=(N !)^{-1} \sum_{\pi \in S_{N}} \Phi(\pi \underline{X}) .
$$

One easily checks that $\Phi_{\sigma}$ is automatically orthogonal in $\otimes^{N} L^{2}\left(\mathbf{R}^{3}\right)$ to the function $\Phi_{\alpha} \equiv \Phi-\Phi_{\sigma}$. For the same reason (symmetrizing the integrand in (2.10), (2.11)) $E_{\Phi}$ splits as $E_{\Phi}=E_{\Phi_{\alpha}}+E_{\Phi_{\sigma}}$. Applying this construction to our $\psi$ we easily conclude that

$$
\begin{aligned}
\left\|\left|\psi_{\sigma}\right|\right\|_{2}^{2} & =(N !)^{-2} \sum_{\mu, \pi \in S_{N}}(|\psi|(\mu \cdot),|\psi|(\pi \cdot)) \\
& \geq(N !)^{-2} \sum_{\pi \in S_{N}}\|\psi\|_{2}^{2}=(N !)^{-1}\|\psi\|_{2}^{2}=(N !)^{-1} .
\end{aligned}
$$

Let $E$ be the unrestricted infimum and let $E^{\sigma}$ denote the infimum restricted to symmetric functions. Then $E^{\sigma}-E \geq 0$ and

$$
\begin{aligned}
E & =\inf _{\psi} E_{\psi}=\inf _{\psi} E_{|\psi|}=\inf _{\psi}\left\{E_{|\psi|_{\alpha}}+E_{|\psi|_{\sigma}}\right\} \\
& \geq \inf _{\psi}\left\{\left\||\psi|_{\alpha}\right\|_{2}^{2} E+\left\||\psi|_{\sigma}\right\|_{2}^{2} E^{\sigma}\right\} \\
& =\inf _{\psi}\left\{\left(1-\left\||\psi|_{\sigma}\right\|_{2}^{2}\right) E+\left\||\psi|_{\sigma}\right\|_{2}^{2} E^{\sigma}\right\} \geq E+(N !)^{-1}\left[E^{\sigma}-E\right] .
\end{aligned}
$$

Hence $E=E^{\sigma}$ as claimed.

In brief, the imposition of the Pauli exclusion principle raises $E$. The miracle is that it raises $E$ enough so that stability of the second kind holds. While it is easy to state that $\psi$ must be antisymmetric (here we return to $\bigwedge^{N} L^{2}\left(\mathbf{R}^{3}\right)$ for simplicity) it is not easy to quantify the effect of antisymmetry. Even the experts have difficulty, for it is not easy to think of an antisymmetric function of a large number of variables.

The most dramatic effect of antisymmetry concerns $T_{\psi}$. In Part III we used the Sobolev inequality to bound $T_{\psi}$ in terms of 
$\rho_{\psi}(x)=|\psi(x)|^{2}$ and we would like now to find a similar bound in the $N$-particle case. It is not useful to define $\rho_{\psi}(\underline{X})=|\psi(\underline{X})|^{2}$ because this is a function of $N$ variables and is quite unmanageable. A more useful definition is the one-particle density $\rho_{\psi}: \mathbf{R}^{3} \rightarrow \mathbf{R}^{+}$ given by

$$
\rho_{\psi}(x)=N \int_{\mathbf{R}^{3(N-1)}}\left|\psi\left(x, x_{2}, \ldots, x_{N}\right)\right|^{2} d x_{2} \ldots d x_{N},
$$

from which it follows that $\int_{\mathbf{R}^{3}} \rho_{\psi}=N$ and $\rho_{\psi}(x)$ is the density of electrons at $x \in \mathbf{R}^{3}$. (Note that, since $\psi$ is antisymmetric, $|\psi|^{2}$ is symmetric and therefore it is immaterial which variable is set equal to $x$ in (4.5).)

It is an easy exercise using Minkowski's inequality to deduce from (3.2) that

$$
T_{\psi} \geq S\left\|\rho_{\psi}\right\|_{3}
$$

in the $N$-particle case, but this inequality is not very useful; it does not distinguish the fact that $\psi$ is antisymmetric instead of symmetric. The right side of (4.6) has the following property. Suppose $\rho$ is a smooth nonnegative function on $\mathbf{R}^{3}$ with $\int \rho=1$ and suppose also that $\rho_{\psi}$ for an $N$ particle $\psi$ is $N \rho$. Then the right side of (4.6) is proportional to $N$. Without the imposition of antisymmetry nothing more can be asserted, but with it $T_{\psi}$ grows like $N^{5 / 3}$, as Theorem 1 below shows.

Let us return to (3.7), the weakened form of Sobolev's inequality for one variable. Again, Minkowski's inequality can be used to translate (3.7) into an inequality for $N$ variables:

$$
T_{\psi} \geq N^{-2 / 3} K^{1} \int_{\mathbf{R}^{3}} \rho_{\psi}(x)^{5 / 3} d x .
$$

The right side of (4.7) also shows a linear dependence on $N$ when $\rho_{\psi}=N \rho$. But antisymmetry comes to the rescue in the form of the following inequality of Lieb and Thirring [LT1, LT2, L1, L2].

Theorem 1 (The 5/3 law for the kinetic energy of fermions). There is a universal constant $K$ such that for all $N$ and all antisymmetric (complex) $\psi \in L^{2}\left(\mathbf{R}^{3 N}\right)$ with $\|\psi\|_{2}=1$

$$
T_{\psi} \geq 2^{2 / 3} K \int_{\mathbf{R}^{3}} \rho_{\psi}(x)^{5 / 3} d x .
$$

If $\psi$ is an antisymmetric function in $L^{2}\left(\mathbf{R}^{3 N} ; \mathbf{C}^{2^{N}}\right)$ then

$$
T_{\psi} \geq K \int_{\mathbf{R}^{3}} \rho_{\psi}(x)^{5 / 3} d x .
$$

$$
K=(2.7709) 2^{-2 / 3}=1.7455 \text { will do. }
$$


Contrast (4.9) with (4.7); the $N^{-2 / 3}$ is gone. The poetic remark at the end of Part III is still correct for many fermions, but $\rho_{\psi}$ is now the total density which, very heuristically, is $N$ times the density of one particle.

Remarks. (1) The sharp $K$ is not known; it is conjectured [LT2] to be $K^{c}=3\left(3 \pi^{2}\right)^{2 / 3} / 5=5.7425$. This number is the "classical" value of $K$ and it arises in the following way. Take a cube $\Gamma_{L} \subset \mathbf{R}^{3}$ of length $L$ and then compute $\tau(n, L)$, the minimum of $T_{\psi}$ over all antisymmtric $n$-particle $\psi$ 's with support in $\left(\Gamma_{L}\right)^{n}$. The best $\psi$ has the form (4.10) below in which each $\phi_{i}$ satisfies $-\Delta \phi_{i}=$ $\lambda_{i} \phi_{i}$ in $\Gamma_{L}$ and $\phi_{i}=0$ on the boundary of $\Gamma_{L}$. One finds, for large $n$, that $\tau(n, L) \approx 2^{2 / 3} K^{c}$ (resp. $K^{c}$ ) times $n^{5 / 3} / L^{2}$ and $\rho_{\psi}(x) \approx n / L^{3}$ for $x \in \Gamma_{L}$ and $\rho_{\psi}(x)=0$ for $x \notin \Gamma_{L}$. These values of $T_{\psi}=\tau(n, L), \rho_{\psi}$ and $K^{c}$ then give equality in (4.8) and (4.9).

(2) Theorem 1 is stated above for the case in which $n$ (the dimension of each variable) is 3 . A similar theorem holds for all $n \geq 1$ (unlike Sobolev's inequality which holds only when $n \geq 3$ ) if $5 / 3$ is replaced by $1+2 / n$ and if $K$ is replaced by a constant $K_{n}$ depending on $n$ (but not on $N$ ). See [LT2 and L2].

To explore the significance of Theorem 1 , and also the significance of the word "exclusion", let us examine the simplest kind of antisymmetric $\psi$, namely a determinantal function. Let $\phi_{1}, \ldots, \phi_{N}$ be any set of $N$ orthonormal functions in $L^{2}\left(\mathbf{R}^{3}\right)$ and let

$$
\psi(\underline{X})=(N !)^{-1 / 2} \operatorname{det}\left\{\phi_{i}\left(x_{j}\right)\right\}_{i, j=1}^{N} .
$$

This $\psi$ is antisymmetric and has $\|\psi\|_{2}=1$. The word "exclusion" comes from the fact that the $\phi_{i}$ 's have to be distinct-in fact orthogonal to each other. The right side of $(4.10)$ is the closest we can come to thinking in terms of "one $L^{2}\left(\mathbf{R}^{3}\right)$ function for each electron". When a physicist or chemist uses a determinantal $\psi$ in calculations the $\phi_{i}$ 's are called orbitals, and one says that "only one electron can be in each orbital". (In the case of $L^{2}\left(\mathbf{R}^{3} ; \mathbf{C}^{2}\right)$ one says that it is possible to have "at most two electrons in each orbital".) In fact it was on the basis of this simple heuristic applied to Bohr's "old quantum mechanics" that Pauli [P] was able to explain the periodic table of the elements in 1925before Schrödinger invented his equation and hence before the relation between antisymmetry and "exclusion" was understood. 
For the $\psi$ given by (4.10), the $\rho_{\psi}$ in (4.5) and $T_{\psi}$ are easily computed to be

$$
\begin{aligned}
\rho_{\psi}(x) & =\sum_{i=1}^{N}\left|\phi_{i}(x)\right|^{2}=\sum_{i=1}^{N} \rho_{\phi_{i}}(x) \\
T_{\psi} & =\sum_{i=1}^{N} \int_{\mathbf{R}^{3}}\left|\nabla \phi_{i}(x)\right|^{2} d x=\sum_{i=1}^{N} T_{\phi_{i}} .
\end{aligned}
$$

For each $\phi_{i},(3.7)$ says that

$$
\int_{\mathbf{R}^{3}}\left|\nabla \phi_{i}(x)\right|^{2} d x \geq K^{1} \int_{\mathbf{R}^{3}}\left|\phi_{i}(x)\right|^{10 / 3} d x .
$$

As an illustration of Theorem 1, consider the case that $\left|\phi_{i}(x)\right|^{2}=$ $\rho(x)$ for each $i$, where $\rho$ is a given nonnegative function with $\int \rho=1$. We could take $\phi_{1}(x)=\rho(x)^{1 / 2}$, but then the remaining $\phi_{j}$ 's would have to be $\phi_{j}(x)=\rho(x)^{1 / 2} \exp \left[i \theta_{j}(x)\right]$, with the $\theta_{j}$ 's real and chosen to insure orthogonality. As $N$ increases, the $\theta_{j}$ 's have to "wiggle" more and more to insure this orthogonality, so that $T_{\phi_{j}}$ increases with $j$. How fast? According to Theorem 1, with $\rho_{\psi}=N \rho$ in this case,

$$
T_{\psi} \geq K N^{5 / 3} \int_{\mathbf{R}^{3}} \rho(x)^{5 / 3} d x
$$

and this shows that $T_{\phi_{j}}$ increases with $j$ like $j^{2 / 3}$, on the average.

Armed with Theorem 1, let us return to the question of finding a lower bound to $E$; this time the $B$ term will not be ignored. Using (4.9) we have for any admissible $\psi$,

$$
\begin{aligned}
E_{\psi} \geq & K \int_{\mathbf{R}^{3}} \rho_{\psi}(x)^{5 / 3} d x+\int W(\underline{X})|\psi(\underline{X})|^{2} d x_{1} \cdots d x_{N} \\
= & K \int_{\mathbf{R}^{3}} \rho_{\psi}(x)^{5 / 3} d x-z \int_{\mathbf{R}^{3}}|x|^{-1} \rho_{\psi}(x) d x \\
& +\int B(\underline{X})|\psi(\underline{X})|^{2} d x_{1} \cdots d x_{N} .
\end{aligned}
$$

Note that the $A$ term of (1.4) is simply expressible in terms of $\rho_{\psi}$, as in (4.15). The $B$ term is more complicated. One would guess by analogy with a fluid of density $\rho_{\psi}$ that roughly

$\int B(\underline{X})|\psi(\underline{X})|^{2} d x_{1} \cdots d x_{N}=\frac{1}{2} \int_{\mathbf{R}^{3}} \int_{\mathbf{R}^{3}} \rho_{\psi}(x) \rho_{\psi}(y)|x-y|^{-1} d x d y$. 
This is not exactly correct, of course, but it can be proved [LO] that for every normalized $\psi$ (with or without the Pauli exclusion principle), the left side minus the right side of (4.16) is bounded below by-(1.68) $\int \rho_{\psi}^{4 / 3}(x) d x$. This error term is small; in any case it can be bounded using the Schwarz inequality by $N^{1 / 2}\left(\int \rho_{\psi}^{5 / 3}\right)^{1 / 2}$ and this can easily be controlled by the kinetic energy term $\int \rho_{\psi}^{5 / 3}$. Therefore, up to controllable errors

$$
E_{\psi} \geq \mathscr{E}^{T F}\left(\rho_{\psi}\right)
$$

where

$$
\begin{aligned}
\mathscr{E}^{T F}(\rho)= & K \int_{\mathbf{R}^{3}} \rho(x)^{5 / 3} d x-z \int_{\mathbf{R}^{3}}|x|^{-1} \rho(x) d x \\
& +\frac{1}{2} \int_{\mathbf{R}^{3}} \int_{\mathbf{R}^{3}} \rho(x) \rho(y)|x-y|^{-1} d x d y .
\end{aligned}
$$

This functional, $\mathscr{E}^{T F}$, which is defined for all nonnegative $\rho$ in $L^{5 / 3}\left(\mathbf{R}^{3}\right) \cap L^{1}\left(\mathbf{R}^{3}\right)$, is called the Thomas-Fermi energy functional. It was introduced independently by Thomas [T] and Fermi [FE] shortly after Schrödinger's discovery, but with the constant $K$ replaced by $K^{c}=3\left(3 \pi^{2}\right)^{2 / 3} / 5$ which, as remarked above, is conjectured to be the sharp constant in Theorem 1 . They were, of course, unaware of Theorem 1 which was proved only in 1975, but they proposed that the Thomas-Fermi ground state energy

$$
E^{T F}=E^{T F}(N, z) \equiv \inf \left\{\mathscr{E}^{T F}(\rho): \int \rho=N\right\}
$$

should be a good approximation to $E$. We have seen that, apart from minor errors, $E^{T F} \leq E$ when $K$ is used instead of $K^{c}$.

The minimization problem (4.18) is an interesting problem in itself and has been analyzed in great detail [LS, L3]. Note that it is defined for all $N \geq 0$, not just integral $N$. A simple scaling $\rho(x) \rightarrow z^{2} \widetilde{\rho}\left(z^{1 / 3} x\right)$ replaces $z$ by $1, N$ by $N / z$ and $E^{T F}(N, z)$ $=z^{7 / 3} E^{T F}(N / z, 1)$. It turns out that the absolute minimum of $\mathscr{E}^{T F}(\rho)$ without regard to the value of $N$ always occurs when $N=z$, i.e., for the neutral atom. There is a unique minimizing $\rho^{T F}$ for (4.18) if $N \leq z$ and there is no minimizer if $N>z$. In either case

$$
\left.E^{T F}(N, z) \geq E^{T F}(z, z)=- \text { (const. }\right) z^{7 / 3}
$$

with (const.) $=-E^{T F}(1,1)>0$.

The remarks in the preceding paragraph apply only to the TF energy and not to the Schrödinger energy but, since $E>E^{T F}$ 
(with $K$ ) to within small errors, we have that the energy of a large atom is bounded below by - (const.) $z^{7 / 3}$. That this is indeed the correct power law was shown by Lieb and Simon [LS]. See also [L3], where a simpler proof is given.

Theorem 2 (TF Theory is asymptotically exact). Fix any $0<\lambda \leq 1$ and let $z_{N}=N / \lambda$ for $N=1,2,3, \ldots$ Let $E\left(N, z_{N}\right)$ be the Schrödinger ground state energy (2.13) for an atom with nuclear charge $z_{N}$ and $N$ electrons and let $\rho_{N}(x)$ in (4.5) be the density for any ground state $\psi_{N}$. Let $E^{T F}\left(N, z_{N}\right)$ and $\rho_{N}^{T F}$ be the corresponding Thomas-Fermi energy and density with $K=K^{c}$ (not the smaller $K$ of Theorem 1). Then

$$
\begin{aligned}
\lim _{N \rightarrow \infty} & E\left(N, z_{N}\right) / E^{T F}\left(N, z_{N}\right) \\
\quad= & \lim _{N \rightarrow \infty} E\left(N, z_{N}\right) /\left[z^{7 / 3} E^{T F}(\lambda, 1)\right]=1 .
\end{aligned}
$$

In the sense of weak $L^{1}\left(\mathbf{R}^{3}\right)$ convergence on compact sets,

$$
\lim _{N \rightarrow \infty} z^{-2} \rho_{N}\left(z^{-1 / 3} x\right)=\lim _{N \rightarrow \infty} z^{-2} \rho_{N}^{T F}\left(z^{-1 / 3} x\right)=\tilde{\rho}(x)
$$

where $\tilde{\rho}$ is the TF minimizer for $z=1$ and $N=\lambda$.

If $\lambda>1$ is fixed and $z_{N}=N / \lambda$ then (4.20) and (4.21) hold with the following replacements:

$$
\begin{aligned}
& E^{T F}\left(N, z_{N}\right) \rightarrow E^{T F}\left(z_{N}, z_{N}\right), \\
& E^{T F}(\lambda, 1) \rightarrow E^{T F}(1,1), \quad \rho_{N}^{T F} \rightarrow \rho_{z_{N}}^{T F}
\end{aligned}
$$

and $\tilde{\rho}$ is the minimizer for $z=1$ and $\lambda=1$. In other words, the Schrödinger quantities for an atom with net negative charge converge to the corresponding TF quantitites for a neutral atom.

From these considerations we can conclude

(1) The energy of a large, neutral atom grows with $z$ like $z^{7 / 3}$. This is significantly different from the earlier result (4.3) without the Pauli exclusion principle because it means that the average energy of each electron is $-z^{4 / 3}$ instead of $-z^{2}$.

(2) For large $z$ almost all of the electrons are located at a distance $z^{-1 / 3}$ from the nucleus. The average electron density in this ball of radius $z^{-1 / 3}$ is $z^{2}$. Without the Pauli exclusion principle we found the distance to be $z^{-1}$ and the electron density to be $z^{4}$.

Conclusion (2) is that even with the Pauli exlcusion principle a large $z$ atom is smaller than a small $z$ atom. This seems to 
contradict the experimental fact that "The large range of atomic masses is not accompanied by a correspondingly large or systematic variation in size. Atomic radii all lie between $0.5 \times 10^{-8} \mathrm{~cm}$ and $2.5 \times 10^{-8} \mathrm{~cm}$, with no marked increase from the lightest to the heaviest [FR]." While it is not possible to give an unambiguous definition of the radius of something as fuzzy as an atom, it appears $[\mathrm{AI}]$ that the largest atom is cesium $(z=55)$ and not uranium $(z=92)$.

The paradox can be resolved by a closer examination of the meaning of "atomic size". If we define the radius to be the radius of the ball in which most of the electrons reside then $z^{-1 / 3}$ is, indeed, the correct answer. But that is not the radius that the chemist sees or the distance between atoms in a solid. This "chemical radius" can be defined, for example, as the radius $R$ such that

$$
\int_{|x|>R} \rho_{\psi}(x) d x=\frac{1}{4}
$$

for a ground state $\psi$. In Thomas-Fermi theory this radius is independent of $z$ for large $z$ (because for large $|x|, \rho^{T F}(x)=$ $C|x|^{-6}$ when $z=N$, with $C$ independent of $z$ ). Theorem 2 (4.21) tells us what $\rho_{N}$ looks like on distance scales of order $z^{-1 / 3}$ where $\rho_{N}$ is of the order $z^{2}$, but it says nothing about distance scales of order one where $\rho_{N}$ is presumably of order one. Nothing is known rigorously about this latter distance scale (and even numerical calculations are uncertain here), but it is surely the case that the true density $\rho_{N}$ in an atom with $z=N$ and with $z$ large looks like that shown very schematically in Figure 1. Also shown in Figure 1 is the innermost region of radius $z^{-1}$. Thomas-Fermi theory states that $\rho^{T F}(x)=$ (const.) $z^{3}$ when $|x|=z^{-1}$ and $\rho^{T F}(0)=\infty$. It is unproved, but undoubtedly true, that when $z$ is large $\rho(x)=C z^{3}[1-O(z|x|)]$ for $0 \leq|x| \leq z^{-1}$, and there is even a conjecture about the precise value of $C$. The investigation of the inner and outer parts of large atoms is now a subject of active research.

Using poetic license again, we can say:

A large atom is like a galaxy. It has a small, high density, energetic core in which most of the electrons are to be found and whose size decreases with $z$ like $z^{-1 / 3}$. The electron-electron electrostatic repulsion always manages to push a few electrons out to a distance of order one which is roughly independent of $z$; this is the radius one observes if one tries to "touch" an atom. 


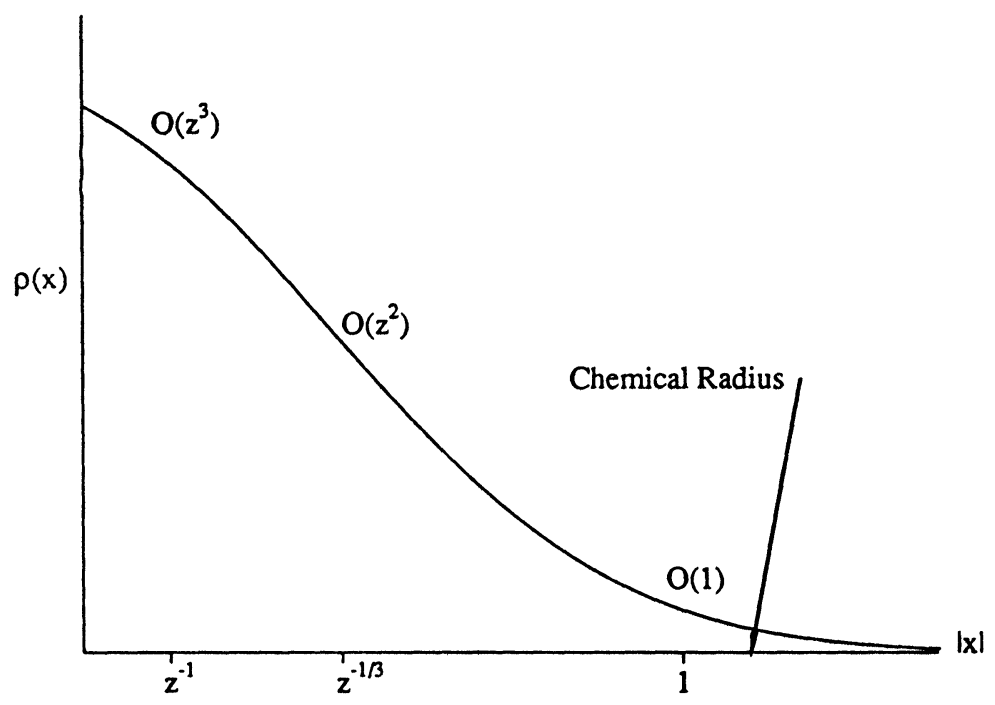

FIGURE 1.

The electron density $\rho(x)$ for a large $z$ atom is crudely plotted as a function of $|x|$, the distance from the nucleus. The graph is intended to show three regimes. (i) At distances of order $z^{-1 / 3}, \rho(x)$ is of order $z^{2}$ and most of the electrons are to be found here. (ii) At the very short distance $z^{-1}, \rho(x)$ is of order $z^{3}$. (iii) At distances of order $1, \rho(x)$ is of order 1 and chemistry takes place. These last two assertions have not yet been established rigorously.

\section{PART V. BULK MATTER: MANY ELECTRONS AND MANY NUCLEI}

The analysis given in Part IV can be applied to the general case of $N$ electrons, $k$ nuclei with charges $\underline{Z}$ and locations $\underline{R}$, and with the full potential energy $W(\underline{X})$ in (1.4). The goal is to prove stability of the second kind: $E(N, k, \underline{Z}) \geq-A(z)(N+k)$ for a suitable $z$-dependent constant $A(z)$, cf. (2.14). This is the theorem first proved by Dyson and Lenard [DL, LE], but with $A(z)$ unrealistically large-of the order $10^{14} z^{2}$.

Before giving the proof let us discuss the crucial importance of this linear law. For simplicity, assume that all the $z_{j}$ 's have the same value $z$ and that $N=k z$, i.e., that the system is neutral. First of all, by a suitable choice of a comparison function $\psi$ in 
(2.13) on the one hand, and using the linear lower bound plus Newton's theorem on the other hand, Lebowitz and Lieb proved the following [LL].

\section{Theorem 3 (The existence of the thermodynamic limit).}

There is a number $A^{\prime}(z)$ such that

$$
\lim _{N \rightarrow \infty} N^{-1} E(N, k=N / z, Z)=-A^{\prime}(z) \text {. }
$$

The linear law is not just a lower bound it is, indeed, the correct asymptotic law. This fact is crucial for the following argument. (There are historical and physical reasons that the words thermodynamic limit instead of asymptotic law are used to describe Theorem 3.)

Suppose now that we have two large collections of $N$ electrons and $k=N / z$ nuclei which are far apart from each other. This means that the nuclear coordinates $\underline{R}=\left(R_{1}, \ldots, R_{2 N}\right)$ satisfy $\left|R_{i}-R_{N+j}\right|>d$ for all $i, j \in\{1, \ldots, N\}$ and where $d$ is some very large number. Think of two well separated stones. The ground state energy would then be nearly $2 E(N)$ where $E(N) \equiv$ $E(N, k, Z)$. If the two stones are allowed to come together, the ground state energy becomes $E(2 N)$ and the energy released is $\delta E=2 E(N)-E(2 N)$. With the linear law in the form of Theorem 3, $\delta E=o(N)$; in fact it turns out that $\delta E \sim N^{2 / 3}$, which is the attractive surface energy that makes the two stones eventually adhere to each other. If, on the other hand, $E(N)$ is proportional to $-N^{p}$ with $p>1$ then $\delta E=-\left(2^{p}-2\right) E(N)$. Thus, the released energy would be of the order of the energy in each stone which, using the actual physical value of $E(N)$, is more than 1000 times larger than the energy released in an explosion of TNT of the same mass. As we shall see in Part VI, hypothetical matter composed solely of bosons would have $E(N) \approx-N^{7 / 5}$. Using this value of $E(N)$ instead of the energy in a physical stone enhances the energy by a factor $N^{2 / 5}$; the energy in a smallish stone, $N=10^{23}$, would then be that of a hydrogen bomb [D]. Such "matter" would be very unpleasant stuff to have lying around the house.

A somewhat less dramatic, but more profound way to state the importance of the thermodynamic limit, Theorem 3 , is that it is part of the foundation of thermodynamics [LL]. If this $N \rightarrow \infty$ limit did not exist the argument in the preceding paragraph shows that matter would not behave the way we expect it to behave-even if stability of the second kind is assumed to hold.

Returning to the problem of proving stability of the second kind, we can use Theorem 1 together with the replacement (4.16) (which 
yields a controllable error) to deduce that $E(N, k, \underline{Z}, \underline{R}) \geq$ $E^{T F}(N, k, \underline{Z}, \underline{R})$ when $E^{T F}$ is given by (4.18) but now

$$
\begin{aligned}
\mathscr{E}^{T F}(\rho)= & K \int_{\mathbf{R}^{3}} \rho(x)^{5 / 3} d x-\int_{\mathbf{R}^{3}} V(x) \rho(x) d x \\
& +\frac{1}{2} \int_{\mathbf{R}^{2}} \int_{\mathbf{R}^{3}} \rho(x) \rho(y)|x-y|^{-1} d x d y+U .
\end{aligned}
$$

The Thomas-Fermi functional in (5.1) is like that in (4.17) except that $z|x|^{-1}$ is replaced by $V(x)$ and $U$ is added (cf. (1.4)-(1.8)). These depend on $\underline{Z}$ and $\underline{R}$.

A lower bound for $E^{T \bar{F}}$ can be obtained by omitting the condition that $\int \rho=N$. The absolute minimum occurs [LS, L3] for the neutral case

$$
N=\sum_{j=1}^{k} z_{j}
$$

as it did for the atom in (4.17). One can struggle with minimizing (5.1) or, preferably, one can use a theorem of Teller (see [LS, L3]) which states that atoms do not bind in Thomas-Fermi theory. For arbitrary $N$, this means that there are $k$ positive numbers $N_{1}, \ldots, N_{k}$ with $\sum_{j=1}^{k} N_{j}=N$ such that

$$
E^{T F}(N, k, \underline{Z}, \underline{R}) \geq \sum_{j=1}^{k} E_{\text {atom }}^{T F}\left(N_{j}, z_{j}\right),
$$

where $E_{\text {atom }}^{T F}$ is the solution to the atomic TF minimization problem (4.18). In particular, under the assumption of neutrality (5.2), the optimum choice is $N_{j}=z_{j}$ and thus

$$
E^{T F}\left(\sum_{j=1}^{k} z_{j}, k, \underline{Z}, \underline{R},\right) \geq \sum_{j=1}^{k} E_{\text {atom }}^{T F}\left(z_{j}, z_{j}\right) .
$$

The right side of (5.4) was already computed in (4.19) and therefore, assuming (4.16),

$$
E(N, k, \underline{Z}) \geq- \text { (const.) } \sum_{j=1}^{k} z_{j}^{7 / 3} .
$$

This is our desired goal of stability of the second kind with $A(z)=$ (const.) $z^{7 / 3}$. However, errors were made when (4.16) was used. If these are included and bounded in a straightforward way, it turns 
out that

$$
E(N, k, \underline{Z}) \geq- \text { (const.) }\left\{N+\sum_{j=1}^{k} z_{j}^{7 / 3}\right\},
$$

which also implies stability of the second kind. The constant in (5.6) turns out to be about 5 instead of $10^{14}$ as in [DL].

Incidentally, it should not be inferred that instability of the second kind is necessarily associated with singularities and/or slow fall-off of the potential at infinity - as is the case with the electrostatic potential $|x|^{-1}$. In other words, seemingly "nice" potentials can fail to produce stability of the second kind. Indeed, Thirring [TW, p. 258] shows that if the function $|x|^{-1}$ is replaced everywhere in (1.4)-(1.8) by the seemingly harmless function $(1+2|x|) \exp [-|x|]$ then stability of the second kind does not hold for the modified system.

Having proved stability of the second kind with electrostatic forces, we are left with another question which was stated by Ehrenfest in a 1931 address to Pauli (quoted in [D]):

"We take a piece of metal. Or a stone. When we think about it, we are astonished that this quantity of matter should occupy so large a volume. Admittedly, the molecules are packed tightly together, and likewise the atoms within each molecule. But why are the atoms themselves so big?

Consider for example the Bohr model of an atom of lead. Why do so few of the 82 electrons run in the orbits close to the nucleus? The attraction of the 82 positive charges in the nucleus is so strong. Many more of the 82 electrons could be concentrated into the inner orbits, before their mutual repulsion would become too large. What prevents the atom from collapsing in this way? Answer: only the Pauli principle, 'No two electrons in the same state.' That is why atoms are so unnecessarily big, and why metal and stone are so bulky.

You must admit, Pauli, that if you would only partially repeal your prohibitions, you could relieve many of our practical worries, for example the traffic problem on our streets."

One simple measure of the "size" of our many-body system in a state $\psi$ is the average radius $R_{p}(\psi)$ defined by

$$
\begin{aligned}
{\left[R_{p}(\psi)\right]^{p} } & =\frac{1}{N} \int_{\mathbf{R}^{3}}|x|^{p} \rho_{\psi}(x) d x \\
& =\frac{1}{N} \int \sum_{i=1}^{N}\left|x_{i}\right|^{p}|\psi(\underline{X})|^{2} d x_{1} \cdots d x_{N} .
\end{aligned}
$$


Here, $p$ is any convenient fixed, positive number. According to our experience with ordinary matter, we should expect $R_{p}(\psi)$ to grow with $N$ like $N^{1 / 3}$, i.e., the volume grows like $N$, when $\psi$ is taken to be a ground state for $N$ electrons. Indeed this surmise is correct-in fact it is correct, as will now be proved, even for some $\psi$ 's which are quite far from being ground states [L1] (see also [LT2]).

Assume that the locations of the nuclei and a normalized $\psi$ satisfy only the condition

$$
E_{\psi} \leq 0
$$

which certainly includes the absolute ground states because $E(N, k, \underline{Z})<0$ always. Then

$$
E_{\psi}=\frac{1}{2} T_{\psi}+E_{\psi}^{\prime}
$$

where $E_{\psi}^{\prime}$ is the energy in (2.12) but with $T_{\psi}$ replaced by $\frac{1}{2} T_{\psi}$ (which amounts to doubling the electron mass). It is easy to see, by a simple scaling $\psi(\underline{X}) \rightarrow 2^{3 N / 2} \psi(2 \underline{X})$ and $R_{j} \rightarrow \frac{1}{2} R_{j}$ that $E^{\prime}(N, k, \underline{Z}, 2 \underline{R})=2 E(N, k, \underline{Z}, \underline{R})$ and therefore $E^{\prime}(N, k, \underline{Z})$ $=2 E(N, k, \underline{Z})$. By (5.8) and (5.9) and stability of the second kind

$$
T_{\psi} \leq 4|E(N, k, \underline{Z})| \leq 4 A(z)(N+k) .
$$

An easy general inequality is the existence of a positive constant $C_{p}$ such that

$$
\left\{\int_{\mathbf{R}^{3}} \rho(x)^{5 / 3} d x\right\}\left\{\int_{\mathbf{R}^{3}}|x|^{p} \rho(x) d x\right\}^{2 / p} \geq C_{p}\left\{\int_{\mathbf{R}^{3}} \rho(x) d x\right\}^{5 / 3+2 / p}
$$

for all nonnegative $\rho$. Using the basic kinetic energy inequality (4.9) together with (5.10) and the fact that $\int \rho_{\psi}=N$, we conclude that

$$
4 A(z)(N+k) \geq T_{\psi} \geq K C_{p} N^{5 / 3} R_{p}(\psi)^{-2} .
$$

We do not want to impose neutrality (5.2) but let us assume $N \leq 2 \sum_{j} z_{j}$; one can show [L4] that this is the only case that needs to be treated. Then (5.12) implies that for each $p>0$

$$
R_{p}(\psi) \geq C_{p}^{\prime} N^{1 / 3}
$$

as required. Here $C_{p}^{\prime}$ depends on $p$ and on $z$, the maximum of the nuclear charges. 
In summary, bulk matter is stable, and has a volume proportional to the number of particles, because of the Pauli exclusion principle for fermions (i.e., the electrons). Effectively the electrons behave like a fluid with energy density $\rho_{\psi}^{5 / 3}$, and this limits the compression caused by the attractive electrostatic forces.

\section{PART VI. BOSONS IN BULK}

The previous paragraph states inter alia that the Pauli exclusion principle is essential for stability of the second kind. The verification of this assertion and the dependence of the energy on $N$ without the Pauli principle is itself an interesting mathematical problem. As explained in Part IV, omitting the Pauli principle is equivalent to studying the minimization problem (2.13) for symmetric functions, i.e., bosons.

THE $N^{5 / 3}$ LAW FOR BOSONS. First, let us see what happens if we try to use the Thomas-Fermi energy as a lower bound-as was done in Part V. Theorem 1 is not available for bosons and (4.7) must be used in place of (4.9). The difference between $K^{1}$ and $K$ is not important, but the factor $N^{-2 / 3}$ is crucial. For fixed $N$, the substitution $K \rightarrow N^{-2 / 3} K^{1}$ in (5.1) is mathematically equivalent to replacing the electron mass (which is 1 in our units) by $m^{\prime}=N^{2 / 3} K / K^{1}$. By the scaling $\underline{R} \rightarrow \underline{R} / m^{\prime}$ and $\psi(\underline{X}) \rightarrow\left(m^{\prime}\right)^{3 N / 2} \psi\left(m^{\prime} \underline{X}\right)$ for the Schrödinger theory or $\rho(x) \rightarrow$ $m^{\prime} \rho\left(m^{\prime} x\right)$ in TF theory one easily finds, in both theories, that $E(N, k, \underline{Z}) \rightarrow m^{\prime} E(N, k \underline{Z})$. Thus, using (5.6),

$$
E(N, k, Z) \geq- \text { (const.) } N^{2 / 3}\left(N+\sum_{j=1}^{k} z_{j}^{7 / 3}\right)
$$

for bosons.

This certainly violates stability of the second kind. If we take $z_{j}=z$ and $N k=z$ as before then $E(N) \geq-$ (const.) $N^{5 / 3}$. Is this result correct or is it simply a bad lower bound? The answer is that the $N^{5 / 3}$ law for bosons with fixed nuclei is correct. It can easily be proved by the following simple choice [L5] of a comparison function $\psi$ for (2.13) and nuclear coordinates $R_{1}, \ldots, R_{k}$.

Let $f: \mathbf{R}^{1} \rightarrow \mathbf{R}$ be given by $f(x)=\sqrt{3 / 2}[1-|x|]$ if $|x| \leq 1$ and $f(x)=0$ otherwise. For each $\lambda>0$ define $\phi_{\lambda}: \mathbf{R}^{3} \rightarrow \mathbf{R}$ by

$$
\phi_{\lambda}\left(x^{1}, x^{2}, x^{3}\right)=\lambda^{3 / 2} f\left(\lambda x^{1}\right) f\left(\lambda x^{2}\right) f\left(\lambda x^{3}\right)
$$


with $x=\left(x^{1}, x^{2}, x^{3}\right)$, so that for all $\lambda$

$$
\left\|\phi_{\lambda}\right\|_{2}=1 \text { and } T_{\phi_{\lambda}}=9 \lambda^{2} \text {. }
$$

The support of $\phi_{\lambda}$ is the cube $\Gamma_{\lambda}=\lambda^{-1} \cdot[-1,1]^{3}$ of volume $8 \lambda^{-3}$. Our comparison function will be

$$
\psi_{\lambda}(\underline{X})=\prod_{i=1}^{N} \phi_{\lambda}\left(x_{i}\right)
$$

so that

$$
\rho_{\psi_{\lambda}}(x)=N \phi_{\lambda}(x)^{2} \text { and } T_{\psi_{\lambda}}=9 N \lambda^{2} .
$$

For this $\psi_{\lambda},(4.16)$ turns out to be nearly exact.

$$
\begin{aligned}
& \int B(\underline{X}) \psi_{\lambda}(\underline{X})^{2} d x_{1} \cdots d x_{N} \\
& \quad=\frac{1}{2} \frac{N-1}{N} \int_{\mathbf{R}^{3}} \int_{\mathbf{R}^{3}} \rho_{\psi_{\lambda}}(x) \rho_{\psi_{\lambda}}(y)|x-y|^{-1} d x d y
\end{aligned}
$$

and the last integral is proportional to $N^{2} / \lambda$. Now assume for simplicity that $N / z$ is an integer and take $k=N / z$ nuclei of charge $z$. It is possible [L5] to place these nuclei in $\Gamma$ (roughly in a periodic arrangement) so that the total potential energy, $W=$ $-A+B+U$, is equal to $-C z^{2 / 3} N^{4 / 3} \lambda$, where $C$ is some positive constant. (To understand this, note that in a volume which is roughly $d^{3}=1 / k \lambda^{3}$ there is one nucleus and $z$ units of negative charge. The total potential energy is then roughly $-k z^{2} / d$.) With these favorable locations of the nuclei,

$$
E_{\psi}=9 N \lambda^{2}-C z^{2 / 3} N^{4 / 3} \lambda .
$$

When this is minimized with respect to $\lambda$, one finds $\lambda=\frac{C}{18} z^{2 / 3} N^{1 / 3}$ and therefore the upper bound

$$
E(N, k, \underline{Z}) \leq-\frac{C^{2}}{36} z^{4 / 3} N^{5 / 3} .
$$

Thus bosonic matter is not only unstable of the second kind but also its radius, $\lambda^{-1}$, decreases with $N$ like $N^{-1 / 3}$. We see here an example of the fact that lack of stability is intimately connected with collapse in the geometric sense.

THE $N^{7 / 5}$ LAW FOR BOSONS. The $N^{5 / 3}$ law, which was derived above, is not the end of the story. Observe that it was crucially important that the nuclei were fixed points that could be 
located at will in space. Suppose we dispense with that approximation and treat the nuclei also as dynamical quantum mechanical particles. Of course they will also be assumed to be bosons, for if they are fermions then we would merely have a charge reversal of the situation described in Part V, i.e., stability of the second kind would hold.

For simplicity we take $z=1$ and assume that these positive bosons also have unit mass. The Hamiltonian is then, with $x_{i}$ denoting the coordinates of the negative bosons and $R_{i}$ denoting the coordinate of the positive bosons,

$$
H_{N, k}=-\sum_{i=1}^{N} \Delta_{x_{i}}-\sum_{j=1}^{k} \Delta_{R_{i}}+W(\underline{X}, \underline{R}) .
$$

The potential function $W(\underline{X}, \underline{R})$ is that given in (1.4)-(1.8) with $e=1$; there it was denoted simply by $W(\underline{X})$. The operator $H_{N, k}$ acts on functions $\psi(\underline{X}, \underline{R})=\psi\left(x_{1}, \ldots, x_{N}, R_{1}, \ldots, R_{k}\right)$ in $\otimes^{N} \otimes^{k} L^{2}\left(\mathbf{R}^{3}\right)$. The ground state energy is given by (2.13), as usual, with $E_{\psi} \equiv\left(\psi, H_{N, k} \psi\right)$. As explained in Part IV, the optimum choice for $\psi$, neglecting symmetry considerations, is always a function that is symmetric in the $x_{i}$ 's and symmetric in the $R_{i}$ 's. But this is precisely the statement that both kinds of particles are bosons.

It is much more difficult to estimate the ground state energy for $H_{N, k}$ in (6.9) than for our previous problem which uses the $H$ in (2.17). The first person to do this rigorously was Dyson [D] who proved the following in 1967.

Theorem 4 (Upper bound for the energy of bosons).

Without loss of generality assume that $N \leq k$. Then the ground state energy, $E(N, k)$, for bosons with the Hamiltonian $H_{N, k}$ satisfies

$$
E(N, k) \leq-1.32 \times 10^{-6} N^{7 / 5}
$$

for large $N$. (It is asserted [D] that this constant can be improved substantially.)

An extended comment is in order here. While the $N^{7 / 5}$ law (6.10) may seem superficially to be only a small improvement over the $N^{5 / 3}$ law, the conceptual difference is enormous. The proof in Part IV of stability of the second kind for fermions (electrons) and fixed positive particles (nuclei) could be understood solely in terms of what is called semi-classical considerations, i.e., ThomasFermi theory. In (5.1) we see that the last three terms on the right 
side are simply the classical electrostatic energy of a charged fluid of density $\rho(x)$ interacting with the fixed nuclei. Once we accept the input from quantum mechanics that the kinetic energy is like that of a fluid with energy density $\rho(x)^{5 / 3}$, the whole energy has a simple classical interpretation-in other words the one-particle density $\rho(x)$ determines the energy with reasonable accuracy.

On the contrary, Theorem 4 cannot be understood this way. In order to achieve an energy as low as $-N^{7 / 5}$, intricate correlations between the positive and negative particles have to be built into a comparison $\psi(\underline{X}, \underline{R})$, and then the potential energy cannot be expressed solely in terms of $\rho_{\psi}$. A delicate balance between potential and kinetic energies is needed and, in the end, it is impossible to think of (6.10) in a simple way-although Dyson [D] does try to give a heuristic explanation of (6.10). Dyson's comparison $\psi$ that leads to $(6.10)$ is very complicated and five pages are needed just to compute $E_{\psi}$. This $\psi$ was suggested by work of Bogolubov in 1947 on the superfluidity of liquid helium and it is similar in many ways to the $\psi$ used by Bardeen, Cooper and Schrieffer in their 1957 Nobel prize winning work on superconductivity. Therefore, if the $N^{7 / 5}$ law is really correct, and not just an upper bound, this kind of highly correlated $\psi$ will be validated in some weak sense as a good approximation to the true ground state.

Two decades later the requisite lower bound was proved by Conlon, Lieb and H-T. Yau [CLY]. The proof is too involved to explain here, even heuristically, but the main result is the following.

Theorem 5 (Lower bound for the energy of bosons).

Without loss of generality assume that $N \leq k$. Then the ground state energy $E(N, k)$ for bosons with the Hamiltonian $H_{N, k}$ satisfies

$$
E(N, k) \geq-A N^{7 / 5}
$$

for some universal constant $A$. If $N=k$ (neutrality) and $N$ is large then $A$ can be taken to be 0.79 in (6.11).

\section{PART VII. RELATIVISTIC MATTER}

According to Einstein's 1905 special theory of relativity, the relativistic kinetic energy as a function of the momentum $p \in \mathbf{R}^{3}$ should be

$$
T(p)=\sqrt{p^{2} c^{2}+m^{2} c^{4}}-m c^{2}
$$


instead of $p^{2} / 2 m$ as in (1.11). Here $c$ is the speed of light. The quantity $m c^{2}$ is called the rest energy and it has been substracted off in (7.1). If $p^{2} \ll m c^{2}$ then $T(p) \approx p^{2} / 2 m$ as before. Using the first part of (1.13) the relation between $p$ and the velocity $v$ is

$$
v=p c^{2}\left(p^{2} c^{2}+m^{2} c^{4}\right)^{-1 / 2} .
$$

When $p^{2} \ll m c^{2}$ this gives $v \approx p / m$ as before, but if $p^{2} \rightarrow \infty$ then $v^{2} \rightarrow c^{2}$. Thus no particle can move faster than the speed of light, and a measure of the importance of relativistic effects is the ratio $|v| / c$.

To gain an understanding of the possible effect of relativistic mechanics on atoms it is convenient to introduce the number

$$
\alpha=e^{2} / \hbar c \text {. }
$$

This is a dimensionless constant whose numerical value is $1 / 137$ and it is called the fine structure constant. (The reason for this appelation is that $\alpha$ also governs certain small effects in atoms that are connected with the electron spin and magnetism, and that have not been discussed here; these effects cause a small splitting of each spectral line into several nearby lines-that experimentalists call the fine structure of the line.)

What is the value of $|v| / c$ for an electron in a hydrogenic atom? We can take $v^{2}$ in a state $\psi$ to be $(2 / m)$ times the kinetic energy $T_{\psi}$ (which is $p^{2} / 2 m$ classically). Using the ground state $\psi$ given by (3.6), and restoring all units (recall that we took $\hbar^{2} / 2 m=1$ and $e=1$ ), one easily finds from the definition (2.6) that

$$
|v| / c=\alpha z \text {. }
$$

For hydrogen $(z=1),|v| / c=1 / 137$ so relativistic effects are unimportant. But when $z$ is large (7.4) correctly gives $|v| / c$ for the "innermost" electrons, and we see that it is not small.

The problem of combining relativistic mechanics with quantum mechanics is an old and extremely difficult one. It is not even easy at the classical mechanics level. At that level one could do the following. Return to the classical Hamiltonian function in (1.11) and simply replace $p_{i}^{2} / 2 m$ by $T\left(p_{i}\right)$ and then use the equations of motion (1.13). While such a dynamical theory makes sense mathematically, it is not a relativistic theory. The reason is that it is not invariant under Lorentz transformations of space-time unless $W \equiv 0$. To remedy this defect it is necessary to give up the idea of particles interacting instantaneously by a force (1.9) which 
depends only on their locations in $\mathbf{R}^{3}$. Instead, it is necessary to invoke the intervention of the full electromagnetic field, to introduce a piece of the Hamiltonian for this field (whose equations of motion in the spirit of (1.13) turn out to be Maxwell's equations), and to introduce another piece of the Hamiltonian which gives the interaction of the particles with the field. All this is very complicated and the final equations of motion do not even make strict mathematical sense for point particles.

The situation is even worse when quantum mechanics is introduced because then the electromagnetic field also has to be "quantized." This is the enormous subject of quantum electrodynamics (Q.E.D) which, in turn, is part of an even larger subject-quantum gauge field theory. (Then there is superstring theory which is still more complicated.)

When $\alpha=0$ (equivalently $e=0$ ) Q.E.D. is trivial because then the electromagnetic field is decoupled from the particles and there are no interactions. This suggests that one can solve problems in Q.E.D. by making a power series expansion in $\alpha$ since $\alpha$ is small. Indeed, such a "perturbation theory" has been investigated in great detail and many of its predictions are confirmed incredibly well by experiments. At present, however, nobody knows in what sense this power series converges, if at all, or how to find any except the first few terms in the series, or what a "nonperturbative" theory would predict. In particular, what happens when there is a very large number of electrons and nuclei, in which case a perturbative treatment is inadequate? Does Q.E.D. predict the stability of matter?

It should be stated that the particle aspect of Q.E.D. is not built on the Schrödinger $p^{2}=-\hbar^{2} \Delta$, which is a second order elliptic operator, but instead on the Dirac operator which is a quartet of first order operators which acts on four-component spinors. The Dirac operator is relativistic (i.e., it is invariant under Lorentz transformations), but if we simply add the potential energy term $W(\underline{X})$ to it the theory will still not be relativistic for the same reason as before (i.e., instantaneous interactions are not Lorentz invariant). Nevertheless, this kind of "mixed" theory is often used instead of the Schrödinger Hamiltonian because it presumably takes account of most of the relativistic corrections. However, from our point of view the Dirac Hamiltonian has a much more serious defect: the ground state energy is not given by a minimization problem as in (2.13). In fact the spectrum of the Dirac Hamiltonian is not bounded below and Dirac had to introduce an extra axiom in order to decide which of the many eigenvalues should be considered as 
the ground state energy. (This axiom is known as "filling the negative energy sea;" unfortunately, it is ambiguous in the many-body case. The axiom also led to the prediction of certain kinds of elementary particles called antimatter; the first one to be discovered was the positron (antielectron) by Anderson in 1932.)

In order to investigate the stability of matter with relativistic kinetic energy, $T(p)$, in a mathematically rigorous way, we are led to study the following theory which uses a "relativistic" modification of the Schrödinger energy. This theory is a caricature of the proper theory, but it will have the advantage of being a well-posed minimization problem as before, and one which is tractable-unlike Q.E.D. At present it is the best that can be done to analyze the stability of matter question rigorously.

With $\psi$ as before, replace the kinetic energy of (2.11) by

$$
T_{\psi}=\sum_{i=1}^{N}\left(\psi, T_{o p}\left(p_{i}\right) \psi\right)
$$

where $T_{o p}(p)$ is given by (7.1), but with $p_{i}=-i \hbar \nabla_{x_{i}}$ as before. In other words, $T_{o p}$ is the operator

$$
T_{o p}=\sqrt{-\hbar^{2} c^{2} \Delta+m^{2} c^{4}}-m c^{2}
$$

which is perfectly respectable although, unlike the operator $-\hbar^{2} \Delta / 2 m$, it is not local, i.e., $\left(T_{o p} \psi\right)(x)$ is not determined by $\psi$ in an infinitesimally small neighborhood of $x$. In terms of the Fourier transform $\widehat{\psi}$ given by (2.4) for one particle,

$$
T_{\psi}=\int_{\mathbf{R}^{3}}\left\{\sqrt{p^{2} c^{2}+m^{2} c^{4}}-m c^{2}\right\}|\widehat{\psi}(p)|^{2} d p
$$

replaces (2.6). The definitions (2.12)-(2.14) for the energy are unaltered.

For the purpose of investigating stability, a simplification can be made, and will be made in this part but not in the next. Since

$$
c|p|-m c^{2} \leq T(p) \leq c|p|
$$

the difference of the operators $T_{o p}$ and $c \hbar \sqrt{-\Delta}$ is a bounded operator. Therefore, for both stability of the first and second kind it suffices to replace $T_{o p}$ by the operator $c \hbar \sqrt{-\Delta}$, i.e., we can set $m=0$. Then $T_{\psi}$ becomes, for one particle,

$$
T_{\psi}=c \int_{\mathbf{R}^{3}}|p \| \widehat{\psi}(p)|^{2} d p .
$$


The advantage of this replacement is a scaling invariance that will become apparent in (7.14) and (7.15). It also brings into focus the essential feature of any theory of relativistic quantum mechanics (Q.E.D. or the Dirac equation or the Klein-Gordon equation or whatever) which can be stated as follows. The central fact about quantum mechanics is that $p^{2}$ becomes the operator $-\hbar^{2} \Delta$ and this can be thought of heuristically (as in (3.5)) as the reciprocal of a length squared-the length being essentially the width of a function $\psi$. In nonrelativistic quantum mechanics this operator is also the kinetic energy and it handily controls the potential energy which is proportional to the reciprocal of the same length. In relativistic quantum mechanics, on the other hand, the kinetic energy is essentially $c \sqrt{-\hbar^{2} \Delta}$ and this is only the reciprocal of the length-not the length squared. Thus, both the potential and kinetic energies are on the same footing in a relativistic quantum theory, and we therefore have what would usually be termed "the critical case".

A word about constants is required here. In the nonrelativistic case we used scaling, as stated at the beginning of Part III, to eliminate all constants except for the nuclear charge numbers $z_{j}$. This cannot be done now because $T_{\psi}$ and $W_{\psi}$ scale in the same way. We can, however, use units in which $\hbar c=1$. Then the energy becomes

$$
E_{\psi}=T_{\psi}+\alpha W_{\psi}
$$

where $W_{\psi}$ is given by (2.10) and (1.4) with $e^{2}$ set equal to one there. $T_{\psi}$ is given by

$$
T_{\psi}=\sum_{i=1}^{N}\left(\psi, \sqrt{-\Delta_{x_{i}}} \psi\right)=\int_{\mathbf{R}^{3 N}} \sum_{i=1}^{N}\left|p_{i} \| \widehat{\psi}\left(p_{1}, \ldots, p_{N}\right)\right|^{2} d p_{1} \cdots d p_{N}
$$

with $\widehat{\psi}$ being the $\mathbf{R}^{3 N}$ Fourier transform. The Hamiltonian that replaces $(2.17)$ is

$$
H=\sum_{i=1}^{N} \sqrt{-\Delta_{x_{i}}}+\alpha W(\underline{X})
$$

Thus there are now two constants in the problem: the fine structure constant $\alpha$ and $z$, the maximum nuclear charge. 
Let us begin by analyzing the hydrogenic atom. In this case only the combination $z \alpha$ enters because $U=0$. Heuristic considerations, like those in Part III, would lead us to investigate (cf. (3.5))

$$
\min _{L}\left\{\frac{1}{L}-\frac{z \alpha}{L}\right\}
$$

as an approximation to $E$. This quantity is zero if $z \alpha \leq 1$ and $-\infty$ if $z \alpha>1$. Indeed this conclusion is qualitatively correct becuase of an inequality of Kato $[\mathrm{K}]$ and Herbst $[\mathrm{H}]$ (see also [LY1])

$$
\int_{\mathbf{R}^{3}}|p \| \widehat{\psi}(p)|^{2} d p \geq \frac{2}{\pi} \int_{\mathbf{R}^{3}}|x|^{-1}|\psi(x)|^{2} d x
$$

in which $2 / \pi$ is the sharp constant. Consequently, the hydrogenic atom has the following ground state energy

$$
\begin{aligned}
E & =0, & \text { if } & z \alpha \leq \frac{2}{\pi} \\
& =-\infty & \text { if } & z \alpha>\frac{2}{\pi}
\end{aligned}
$$

and stability of the first kind holds if and only if $z \alpha \leq 2 / \pi$, i.e., $z \leq 87$ with $\alpha=1 / 137$.

Relativistic quantum mechanics stabilizes an atom only if $z \alpha$ is small enough.

It is to be noted that a similar catastrophe occurs with the Dirac Hamiltonian for an atom [KS]. Again, only $z \alpha$ enters and there is a critical value $z \alpha=1$, instead of $2 / \pi$. For $z \alpha \leq \sqrt{3} / 2$ this operator is essentially selfadjoint on $C_{c}^{\infty}$, the infinitely differentiable functions of compact support. When $z \alpha>\sqrt{3} / 2$ the deficiency indices are $(+1,+1)$ and so there is a selfadjoint extension, but only for $\sqrt{3} / 2<z \alpha<1$ is there a distinguished, physical selfadjoint extension. It is distinguished either by analytic continuation from the $z \alpha \leq \sqrt{3} / 2$ case or by finiteness of the potential energy; in other words, when $z \alpha \geq 1$ the kinetic and potential energies cannot be defined separately. When $z \alpha=1$ the hydrogenic ground state energy "falls into the negative energy sea" and the Dirac operator then ceases to make good physical sense. The underlying reason for the catastrophe is the same in both cases: the kinetic energy is $1 / L$ instead of $1 / L^{2}$.

The next step is to investigate large atoms, as in Part VI. Clearly, stability of the first kind holds here if and only if $z \alpha \leq 2 / \pi$ because, as a simple comparison function $\psi(\underline{X})$ demonstrates, the positive electron-electron repulsion $B(\underline{X})$ of $(1.7)$ cannot overcome the $-\infty$ energy to be gained by letting one electron "fall into the nucleus" when $z \alpha>2 / \pi$. 
In fact conclusion (7.14) holds in the large atom case as well. This is where the nice scaling property of $\sqrt{-\Delta}$ and $|x|^{-1}$ come in. To jump ahead for the moment, we can always say, when $m=0$, that in the general case of many electrons and many nuclei there are precisely two alternatives:

$$
\text { either } E(N, k, \underline{Z})=0 \text { or } E(N, k, \underline{Z})=-\infty,
$$

which tells us that stability of the first kind is equivalent to stability of the second kind for relativistic matter. The proof of (7.15) is a simple one: If $E_{\psi}<0$ for some $\psi$ then, by setting $\psi_{\lambda}(\underline{X})=$ $\lambda^{-3 N / 2} \psi(\lambda \underline{X})$, we have that $E_{\psi_{\lambda}}=\lambda E_{\psi}$, and this can be driven to $-\infty$ by letting $\lambda \rightarrow \infty$. If, on the other hand, $E_{\psi}>0$ then we can drive $E_{\psi_{\lambda}}$ to 0 by letting $\lambda \rightarrow 0$.

Apart from the stability question we can also ask about the structure of large atoms-as in Part IV. Is there an appropriate ThomasFermi theory in this case? The answer is No and it is instructive to understand why.

We can start by asking for an analogue of Theorem 1, which is the basic kinetic energy estimate for fermions. There is oneas discovered by Daubechies [DA]: Theorem 1 holds for the $\sqrt{-\Delta}$ kinetic energy if $5 / 3$ is replaced everywhere by $4 / 3$, if $2^{2 / 3}$ is replaced by $2^{1 / 3}$ in (4.8) and if $K$ is changed. ( $K=1.26$ will do.)

So far, so good. Next we form the Thomas-Fermi functional as in (4.17):

$$
\begin{aligned}
\mathscr{E}^{T F}(\rho)= & K \int_{\mathbf{R}^{3}} \rho(x)^{4 / 3} d x-z \alpha \int_{\mathbf{R}^{3}}|x|^{-1} \rho(x) d x \\
& +\frac{1}{2} \alpha \int_{\mathbf{R}^{3}} \int_{\mathbf{R}^{3}} \rho(x) \rho(y)|x-y|^{-1} d x d y .
\end{aligned}
$$

This, unfortunately, is unbounded below for any choice of $N=$ $\int \rho$. (The reader can easily verify that $\int \rho^{4 / 3}$ cannot control the $|x|^{-1}$ singularity, and the last term in (7.16) does not save the situation.) The conclusion is that Thomas-Fermi theory is useless for large atoms (and hence also for the case of many electrons and many nuclei), but in Part VIII we shall see that it is useful for describing the relativistic matter interacting by gravitational forces in a star.

Finally, we turn to bulk matter as in Part V. Now, however, even stability of the first kind is problematic for one electron and many nuclei; it already is problematic even for one electron $(N=1)$ and one nucleus $(k=1)$. The following additional concern now 
presents itself. Suppose there are two nuclei with $z \alpha=3 / 2 \pi$, located at $R_{1}$ and $R_{2}$ in $\mathbf{R}^{3}$, and suppose there is but one electron. If the two points are coincident, say $R_{1}=R_{2}=0$, we have a "bomb." While each nucleus is subcritical the combined nucleus is supercritical, $\widetilde{z} \alpha=3 / \pi$, and the electron can fall into it, releasing an infinite energy (7.15). What prevents this from happening? Answer: the nucleus-nucleus repulsive energy

$$
U=\alpha z^{2}\left|R_{1}-R_{2}\right|^{-1}=\frac{(z \alpha)^{2}}{\alpha}\left|R_{1}-R_{2}\right|^{-1},
$$

which goes to $+\infty$ as $R_{1} \rightarrow R_{2}$ and which is one part of $E(1,2, \underline{Z}, \underline{R})$. There are now genuinely two parameters in the problem, which can conveniently be taken to be $z \alpha$ and $\alpha$. By length scaling, all the energies are proportional to $\left|R_{1}-R_{2}\right|^{-1}$, so $E(1,2, \underline{Z}, \underline{R})=C\left|R_{1}-R_{2}\right|^{-1}$. If $z \alpha$ is fixed at $3 / 2 \pi$, is the constant $1 / \alpha$ in (7.16) large enough so that the repulsion $U$ wins, i.e., so that $C \geq 0$ ?

This exercise with $N=1$ and $k=2$ tells us that stability will require not only an upper bound on $z_{j} \alpha$ of $2 / \pi$ for each nucleus but also an upper bound, $\alpha_{c}$, for $\alpha$. This is clarified in Theorem 6 below. Furthermore, we might fear that this $\alpha_{c}$ goes to zero as $z \alpha \rightarrow 2 / \pi$. Here, $z=\max _{j}\left\{z_{j}\right\}$ as before. It might also happen that $\alpha_{c}$ depends on $N$ and/or $k$. Fortunately, neither of these two fears materializes.

The first result on this problem was by Daubechies and Lieb [DAL] who proved stability for one electron and arbitrarily many nuclei, namely,

$$
E(1, k, \underline{Z})=0
$$

provided $z_{j} \alpha \leq 2 / \pi$ for each $j$ and provided $\alpha \leq 1 / 3 \pi$.

The big breakthrough was by Conlon [CO] who proved for all $N$ and $k$ that

$$
E(N, k, \underline{Z})=0
$$

provided $z_{j}=1$ for all $j$ and provided $\alpha \leq 10^{-200} \quad$ [sic] and provided that spin is omitted, i.e., $\psi \in \bigwedge^{N} L^{2}\left(\mathbf{R}^{3}\right)$ instead of $\psi \in$ $\bigwedge^{N} L^{2}\left(\mathbf{R}^{3} ; \mathbf{C}^{2}\right)$. This was vastly improved by Fefferman and de la Llave [FD] to $\alpha \leq 1 / 2.06 \pi$ with the other conditions remaining the same.

The results of Conlon, Fefferman and de la Llave fall short of the critical case $z \alpha=2 / \pi$. They also do not include all $\psi$ 's because the inclusion of spin is not as easy as it was in the nonrelativistic 
case. The matter was finally settled by Lieb and H-T. Yau [LY1] who treated a slightly more general problem. The method of proof in [LY1] is very different from that in [C] and [FD].

Theorem 6 (Stability of relativistic matter up to $z \alpha=2 / \pi$ ). Let $N$ and $k$ be arbitrary and let $z_{j} \alpha \leq 2 / \pi$ for each $j=1, \ldots, k$. Let $E_{q}(N, k, \underline{Z})$ be the infimum of the energy (7.9) as in (2.11) and (2.14), but with $\psi \in \Lambda_{1}^{N} L^{2}\left(\mathbf{R}^{3} ; \mathbf{C}^{q}\right) . \quad(q=1$ is the simple antisymmetric case, $q=2$ is the physical case and $q>2$ is for fun.) Then $E_{q}(N, k, \underline{Z})=0$ if $q \alpha \leq 1 / 47$. In particular, stability holds in the physical case: $\alpha=1 / 137$.

Is this limitation on $\alpha$ (especially the $1 / q$ dependence) merely an artifact of the proof in [LY1]? What about the stability of relativistic bosonic matter? These questions are answered in the following two theorems [LY1]. Roughly, the proof of Theorem 7 uses the same elementary ideas as in the proof of (6.7) and (6.8).

Theorem 7 (Instability of bulk matter for large $\alpha$ ). With the definition of $E_{q}$ as in Theorem 6, assume there are $N$ electrons and $k$ nuclei, each with the same charge $z>0$.

(1) $q$ and $z$ independent bound. If $\alpha>128 / 15 \pi$, if $N \geq 1$ and if $k \geq 1+1 / z$ then there is collapse for all $q$, i.e., $E_{q}(N, k, \underline{Z})=$ $-\infty$.

(2) $q$ and $z$ dependent bound. If $\alpha>36 q^{-1 / 3} z^{2 / 3}$, if $N \geq q$ and if $k \geq q / z$ then $E_{q}(N, k, \underline{Z})=-\infty$.

Thus, if $\alpha$ is large one electron can cause collapse no matter how small $z$ is, provided enough nuclei are used in order to make the system approximately neutral. When $q$ is large the critical $\alpha$ decreases with $q$ at least as fast as $q^{-1 / 3}$. This contrasts with the $q^{-1}$ dependence of Theorem 6; the exact dependence of the critical $\alpha$ on $q$ is not known.

One reason for mentioning the $q$ dependence is that the choice $q=N$ is equivalent to omitting the Pauli exclusion principle altogether. The interested reader can easily deduce this from the discussion of symmetry in Part IV. This means that the case $q=N$ includes the boson case, and as a corollary of Theorem 7 we have

Theorem 8 (Instability of relativistic bosons with fixed nuclei). Let $\alpha>0$ and assume that all nuclei have a common charge $z>0$. If the dynamic, negative particles are bosons instead of fermions, and if the nuclei are fixed as before, then collapse always occurs for sufficiently large $N$ and $k$, i.e., $E(N, k, \underline{Z})=-\infty$. The choice $N \geq(36)^{3} z^{2} \alpha^{-3}$ and $k \geq(36)^{3} z \alpha^{-3}$ suffices. 


\section{PART VIII. THE STABILITY AND INSTABILITY OF COLD STARS}

Stars shine because they are a vast, continuous nuclear explosion. Nevertheless, the effect of attractive gravitational forces in such a huge body is not negligible-a fact that can easily be appreciated by noting that the sun maintains a spherical shape despite the nuclear violence. The byproducts of the nuclear reactions are light, heat and various kinds of particles, all of which produce an internal pressure that keeps the star expanded before these byproducts can leak out through the surface.

After the nuclear reactions are finished, in what can be called the post-twinkle phase, the star eventually cools, and it might be supposed that it would then collapse because of the gravitational attraction. Sometimes this does happen with great rapidity-for that is what a supernova is; the enormous gravitational energy is then converted into the production of a vast amount of light and numerous particles, mostly neutrinos. But sometimes the cold star, or the remnant of a supernova explosion, which is also a cold star, merely attains a new, much smaller radius and is quite stablealthough lightless. (Another conceivable final state of a supernova is a black hole, in which case the collapse might be silent because black holes do not permit light to escape; I thank F. Dyson for this observation.)

The determining factor for total collapse or stability is the stellar mass; the dividing line is several solar masses. What mechanism is available, in the stable case, to provide the internal pressure that prevents the gravitational attraction from crushing the cold star? Answer: The quantum mechanical kinetic energy of fermions.

Actually there are two kinds of cold stars: neutron stars and white dwarfs. The latter kind will be discussed at the end. The former kind, which are remnants of supernovae, are composed mostly of chargeless neutrons. (In Part I it was stated that a free neutron decays into a proton and an electron in about $13 \mathrm{~min}$ utes, but in the very dense interior of such a star the decay ceases for several reasons.) With $\underline{X}=\left(x_{1}, \ldots, x_{N}\right)$ denoting the coordinates of $N$ neutrons, the classical potential energy is given by Newton's gravitational formula (cf. (1.2) and (1.9))

$$
W(\underline{X})=-\kappa \sum_{1 \leq i<j \leq N}\left|x_{i}-x_{j}\right|^{-1} .
$$

Here, $\kappa=G M_{n}^{2}$, with $G$ being Newton's gravitational constant and $M_{n}$ being the neutron mass (which is just a little bigger than 
the proton mass, $\left.M_{p}\right)$. There is no electrostatic potential energy since the neutron has no electric charge.

Neutron stars were mentioned as a possibility by Landau in 1932, after the discovery of the neutron by Chadwick in the same year [B, ST]. In 1934 Baade and Zwicky proposed their connection with supernovae. Their actual existence was "established" by the 1968 discovery of pulsars (which mostly emit accurately timed pulses of radio waves) and Gold's identification of them as rotating neutron stars. The supposed facts about these stars are amazing [B, ST]. Their mass is a few solar masses (about $10^{57}$ neutrons) but their radius is only about $10 \mathrm{~km}$. Thus the gravitational force at their surface is about $10^{12}$ times that on Earth. The internal structure of such an object is hard to guess, because at these densities-especially the central density-neutrons will cease to look like individual particles and might become some sort of quark soup. Moreover, this large mass in such a small volume will cause space to be "curved" in accordance with the principles of Einstein's general theory of relativity. These complications will be ignored here and we shall suppose that a neutron star is just a collection of $N$ particles with the gravitational potential energy function of (8.1).

The model to be discussed here is standard and it is built along the lines of Part VII. With an abuse of history we shall call it the Chandrasekhar model, for a reason that will become clear at the end. Since the neutron star is cold we can suppose that it is in its quantum mechanical ground state. The energy of a wave function $\psi$ is given, as usual, by $E_{\psi}=T_{\psi}+W_{\psi}$ with $T_{\psi}$ defined in (7.5) with $m=M_{n}$ and $W_{\psi}$ defined in (2.10) with $W(\underline{X})$ given by (8.1). Adopting henceforth units in which $\hbar=c=1$, the Hamiltonian is

$$
H_{N}=\sum_{i=1}^{N}\left\{\sqrt{-\Delta_{x_{i}}+m^{2}}-m\right\}-\kappa \sum_{1 \leq i<j \leq N}\left|x_{i}-x_{j}\right|^{-1} .
$$

with $\kappa=G M_{n}^{2}$ and $m=M_{n}$. The problem is to compute

$$
E(N)=\inf _{\psi} E_{\psi}=\inf _{\psi}\left(\psi, H_{N} \psi\right)
$$

and to delineate the properties of the density $\rho_{\psi}$ for a minimizing $\psi$.

Two things are to be noticed. One is that the neutrons are being treated (special) relativistically with $T(p)=\sqrt{p^{2} c^{2}+m^{2} c^{4}}-$ $m c^{2}$. This is obviously important to do since $|v| / c$ will turn out 
to be quite large at these densities. As in Part VII, the question of collapse or no collapse can be decided by the simpler choice $T(p)=c|p|$, but if we want to compute the density $\rho_{\psi}$ in the stable phase it is essential to keep the full expression (8.2).

The second point is that $W(\underline{X})$ has the same form as the electron-electron repulsion term $B(\underline{X})$ in (1.7) except for one thing-the minus sign. For the repulsive case we were able to approximate this energy in terms of $\rho_{\psi}$ as in (4.16), because, as discussed there, the left side of $(4.16)$ minus the right side is bounded below [LO] by- (1.68) $\int \rho_{\psi}^{4 / 3}$. But because of the minus sign in $W(\underline{X})$ we now require an upper bound for the difference in (4.16) in order to achieve a lower bound to $E_{\psi}$. Unfortunately the only upper bound is $+\infty$. Therefore, controlling $W_{\psi}$ by $T_{\psi}$ is not at all a simple matter in this case.

Nevertheless, there is a simple, well-defined Thomas-Fermi approximation to this problem, as in Part IV, that is obtained using (4.16). Whether it is correct or not remains to be seen. Its construction requires approximating the relativistic kinetic energy (7.5) in terms of $\rho_{\psi}$ in analogy with Theorem 1 or Theorem 2. Imitating the discussion in Remark 1 after (4.9), we place $n$ particles in a cube of size $L$ and compute the minimum of $T_{\psi}$. With $\rho=n / L^{3}$ and $\eta \equiv\left(6 \pi^{2} \rho / q\right)^{1 / 3}$ it turns out to be (for large $n$ ) equal to the volume $L^{3}$ times the quantity

$$
j(\rho)=\frac{q}{2 \pi^{2}} \int_{0}^{\eta}\left\{\left(p^{2}+m^{2}\right)^{1 / 2}-m\right\} p^{2} d p .
$$

(Recall that $q=1$ for $\psi \in \bigwedge^{N} L^{2}\left(\mathbf{R}^{3}\right)$ and $q=2$ for the physical case $\psi \in \Lambda_{1}^{N} L^{2}\left(\mathbf{R}^{3} ; \mathbf{C}^{2}\right)$.) This $j(\rho)$ reduces to $(2 / q)^{2 / 3} K^{c} \rho^{5 / 3}$ when $\rho$ is small (corresponding to the nonrelativistic kinetic energy) and to $\left(3\left(6 \pi^{2}\right)^{1 / 3} / 4 q^{1 / 3}\right) \rho^{4 / 3}$ when $\rho$ is large (corresponding to the relativistic case). It should be remarked that Daubechies [DA], who extended Theorem 1 to $\sqrt{-\Delta}$, as noted before (7.16), also extended it to $\sqrt{-\Delta+m^{2}}-m$.

We can now form the Thomas-Fermi functional for a neutron star.

$$
\mathscr{E}^{T F}(\rho)=\int_{\mathbf{R}^{3}} j(\rho(x)) d x-\frac{\kappa}{2} \int_{\mathbf{R}^{3}} \int_{\mathbf{R}^{3}} \rho(x) \rho(y)|x-y|^{-1} d x d y
$$

and the Thomas-Fermi energy is

$$
E^{T F}(N)=\inf \left\{\mathscr{E}(\rho): \int \rho=N\right\} .
$$


This problem can also be conveniently reformulated by setting

$$
\widetilde{\rho}(x)=\rho\left(N^{1 / 3} x\right) \Rightarrow \int_{\mathbf{R}^{3}} \tilde{\rho}(x) d x=1
$$

so that, with

$$
\tilde{\mathscr{E}}^{T F}(\rho)=\int_{\mathbf{R}^{3}} j\left(\rho(x) d x-\frac{1}{2} \kappa N^{2 / 3} \int_{\mathbf{R}^{3}} \int_{\mathbf{R}^{3}} \rho(x) \rho(y)|x-y|^{-1} d x d y,\right.
$$

the energy becomes

$$
\frac{1}{N} E^{T F}(N)=\inf \left\{\tilde{\mathscr{E}}^{T F}(\rho): \int \rho=1\right\} \equiv e^{T F}\left(\kappa N^{2 / 3}\right) .
$$

The advantage of (8.7)-(8.9) is that it makes clear that the relevant parameter in the problem is $\tau=\kappa N^{2 / 3}$. Numerically, $\kappa=G m_{N}{ }^{2}$ is about $10^{-38}$ in our units and $N \approx 10^{57}$, so that $\tau \approx 1$. This suggests that it is an excellent approximation, indeed, to consider the limit $\kappa \rightarrow 0, N \rightarrow \infty$ but with $\kappa N^{2 / 3}=\tau$ held fixed.

The first attempt [LT3] relate $E(N)$ to $E^{T F}(N)$ succeeded to within a factor of 4 . The following theorem of Lieb and H-T. Yau [LY2] finally settled the question.

Theorem 9 (Thomas-Fermi theory is asymptotically exact for cold stars). Fix $\tau=\kappa N^{2 / 3}$ and let $\kappa \rightarrow 0$ and $N \rightarrow \infty$. Then

$$
\lim _{\kappa \rightarrow 0, N \rightarrow \infty} E(N) / N=e^{T F}(\tau) .
$$

(The error, for finite $N$, can be bounded.) Furthermore, there is a critical number, $\tau_{c}$, such that the Thomas-Fermi minimization problem (8.8), (8.9) satisfies

(1) If $\tau<\tau_{c}$ then $e^{T F}(\tau)$ is finite and there is a unique minimizing $\rho_{\tau}^{T F}$.

(2) If $0<\tau<\tau_{c}$ and if $\psi_{N}$ is a minimizer for the quantum mechanical problem with density $\rho_{\psi_{N}}$ then, weakly in $L^{4 / 3}\left(\mathbf{R}^{3}\right) \cap$ $L^{1}\left(\mathbf{R}^{3}\right)$,

$$
\lim _{\kappa \rightarrow 0, N \rightarrow \infty} \rho_{\psi_{N}}\left(N^{1 / 3} x\right)=\rho_{\tau}^{T F}(x) .
$$

(3) The function $N \mapsto E^{T F}(N)$, for fixed $\kappa$, is concave and decreasing.

(4) If $\tau>\tau_{c}$ then $e^{T F}(\tau)=-\infty$, while $e^{T F}\left(\tau_{c}\right)$ is finite.

Thus, a neutron star is stable if $\kappa N^{2 / 3}<\tau_{c}$ and it collapses if $\kappa N^{2 / 3}>\tau_{c}$. It is not hard to see from (8.5) that such a collapse 
occurs and, indeed, to infer the value of $\tau_{c}$. For large $\rho$ (which is relevant here) $j(\rho)=C \rho^{4 / 3}$ with $C=3\left(6 \pi^{2}\right)^{1 / 3} / 4 q^{1 / 3}$. The sharp inequality

$$
\begin{aligned}
& A\left\{\int_{\mathbf{R}^{3}} \rho(x) d x\right\}^{2 / 3} \int_{\mathbf{R}^{3}} \rho(x)^{4 / 3} d x \\
& \quad \geq \frac{1}{2} \int_{\mathbf{R}^{3}} \int_{\mathbf{R}^{3}} \rho(x) \rho(y)|x-y|^{-1} d x d y
\end{aligned}
$$

is well known, with $A=1.092$. Therefore

$$
\tau_{c}=C / A \text {. }
$$

The Euler-Lagrange equation for the problem (8.8), (8.9) is

$$
j^{\prime}(\rho(x))=\left[\eta(x)^{2}+m^{2}\right]^{1 / 2}-m=\left\{\tau|x|^{-1} * \rho-\mu\right\}_{+},
$$

where $\eta(x)=\left(6 \pi^{2} \rho(x) / q\right)^{1 / 3}$, * denotes convolution, $\mu>0$ is a Lagrange multiplier (which has to be adjusted so that $\int \rho=1$ ) and $\{A\}_{+}=\max (A, 0)$. The optimum solution $\rho$ is spherically symmetric, decreasing and has compact support in a ball $B_{R}$ of radius $R$. The Euler-Lagrange equation can be converted into a differential equation by applying the Laplacian, $\Delta$, to (8.14).

$$
-\Delta \Theta=4 \pi\left[\Theta^{2}+2 \Theta\right]^{3 / 2} \text { in } B_{R}
$$

with $\Theta=\kappa|x|^{-1} * \rho$ and $\Theta(x)=0$ when $|x|=R$.

The existence of a solution to (8.14), (8.15) was proved by Auchmuty and Beals $[\mathrm{AB}]$. The analysis of this equation is, in itself, an interesting mathematical problem. The following has been proved [LY2].

Theorem 10 (Properties of the Thomas-Fermi density). There is exactly one nonnegative radial solution to (8.14) for each $\mu>0$ and $0<\tau<\tau_{c}$. It has compact support. For $\tau \geq \tau_{c}$ there is no solution (even though $e^{T F}\left(\tau_{c}\right)$ is finite). If $\tau<\tau_{c}$ there is exactly one $\mu$ so that $\int \rho=1$. Both $\rho$ and $\mu$ are continuous functions of $\tau$ and $\rho(0)$ is an increasing function of $\tau$, while the radius $R$ is a decreasing function with $R \rightarrow 0$ as $\tau \rightarrow \tau_{c}$ and $R \rightarrow \infty$ as $\tau \rightarrow 0$. If $\tau_{1}<\tau_{2}<\tau_{c}$ then the solutions $\rho_{1}$ and $\rho_{2}$ satisfy $\rho_{1}(x)=\rho_{2}(x)$ for exactly one value of $|x|$.

These properties show that the star has a unique shape that evolves continuously with $\tau$, i.e., there are no jumps.

What would happen if the neutrons, which are fermions, are replaced by bosons? Elementary particles that are bosons, and that are stable have never been seen, as stated earlier, but they 
are posited to exist in certain theories in order to account for the "missing mass" in the universe. They are called axions and are not expected to condense into stars because they cannot get rid of their gravitational energy easily, but it is amusing to consider the possibility anyway. See [RB].

For bosons we might imagine that a simple product function $\psi(\underline{X})=\Pi \phi\left(x_{i}\right)$ with $\phi \in L^{2}\left(\mathbf{R}^{3}\right)$, as in (4.2), would adequately describe the ground state. Then $\rho_{\psi}(x)=N|\phi(x)|^{2}$ and with $\phi(x) \geq 0$ (which is the optimum choice),

$$
\begin{aligned}
T_{\psi} & =N\left(\phi, T_{o p} \phi\right)=\left(\sqrt{\rho_{\psi}}, T_{o p} \sqrt{\rho_{\psi}}\right) \\
W_{\psi} & =\frac{\kappa}{2} \frac{N-1}{N} \int_{\mathbf{R}^{3}} \int_{\mathbf{R}^{3}} \rho_{\psi}(x) \rho_{\psi}(y)|x-y|^{-1} d x d y .
\end{aligned}
$$

Adding these (and supposing $N$ to be large) we obtain a Hartree type functional

$$
\mathscr{E}^{H}(\rho)=\left(\sqrt{\rho}, T_{o p} \sqrt{\rho}\right)-\frac{\kappa}{2} \int_{\mathbf{R}^{3}} \int_{\mathbf{R}^{3}} \rho(x) \rho(y)|x-y|^{-1} d x d y
$$

for nonnegative $\rho$ with $\int \rho=N$. This differs from the ThomasFermi functional in the replacement of $\int j(\rho)$ by (8.16). As usual, we define the Hartree energy to be

$$
E^{H}(N)=\inf \left\{\mathscr{E}^{H}(\rho): \int \rho=N\right\} .
$$

The scaling $\rho(x) \rightarrow N \rho(x)$ reduced the problem to one in which $\int \rho=1, \kappa \rightarrow \kappa N \equiv \omega$ and $E \rightarrow N E$. From this we learn that the parameter $\omega=\kappa N$ is the crucial one for bosons, not $\tau=\kappa N^{2 / 3}$.

The minmization of (8.18) leads now to a genuine differential equation for $f=\sqrt{\rho}$

$$
\left\{T_{o p}-\omega|x|^{-1} * f^{2}\right\} f=-\mu f
$$

on all of $\mathbf{R}^{3}$ and with $\mu$ chosen so that $\int f^{2}=N$. It is proved in [LY2] that again there is a critical value $\omega_{c}$ such that when $\omega<\omega_{c}$ a minimizer exists and it is spherically symmetric and decreasing (although not with compact support). If $\omega>\omega_{c}$ then $E^{H}(N)=-\infty$. If we fix $\omega$ and let $\kappa \rightarrow \infty$ and $N \rightarrow \infty$ with $\omega=\kappa N$ then, as in the previous fermionic case, the solution to the quantum problem converges to the solution to the Hartree problem, i.e., $E(N) / E^{H}(N) \rightarrow 1$ and $\frac{1}{N} \rho_{\psi}-\frac{1}{N} \rho^{H} \rightarrow 0$. 
Thus, bosonic stars can collapse as well, but whereas in the fermionic case the critical number $N_{c}$ is proportional to $\kappa^{-3 / 2} \approx$ $10^{57}$, for bosons $N_{c}$ is proportional to $\kappa^{-1} \approx 10^{38}$. The former defines an object which is the size of a star, while the latter defines only an object as massive as a mountain (assuming that the same value is used for the mass of the constituent particles.)

It is possible to understand this $\kappa^{-1}$ behavior in a simple waywhich also explains the central difficulty in proving Theorem 9. In (7.14) we saw that the kinetic energy of a particle ceases to control a $-C /|x|$ singularity whenever $C>2 / \pi$. In our bosonic star case we have many particles with the tiny constant $C \equiv \kappa$ between each pair. Suppose now that $(N-1) \kappa$ exceeds $2 / \pi$ and that $N-1$ particles come together at a common point. Then the $N^{\text {th }}$ particle feels an over-critical attraction and "falls in the hole." But then every particle is trapped and none can escape. Something like this artificial scenario is what happens in the bosonic case and leads to collapse if $\omega=N \kappa$ is big enough. (It is not claimed that $\omega_{c}=2 / \pi$; in fact it is known only that $4 / \pi<\omega_{c}<2.7$.) The interesting fact about fermions is that the Pauli exclusion principle prevents this scenario from happening. Since $N \approx \kappa^{3 / 2}$ in this case, it would require the coalescence of only a tiny fraction of the particles (namely $N^{2 / 3} / N$ ) in order to form a "trap" for the remaining particles-but such a "fluctuation" does not occur with any significant probability.

Now let us return to the second kind of cold star-the white dwarf. In 1914 Adams discovered that while the companion star of Sirius has a mass about equal to that of the sun (which was known from its perturbation of Sirius) its radius had to be very small [ST]. He inferred this from the fact that the star is hot (in fact its light is whiter than that of most stars-hence the name) but it has a very small total luminosity. Many such stars are now known and they are thought to be burned out stars consisting of ordinary electrons and nuclei such as we discussed in Part VII. Their radius is bigger than that of a neutron star-about 5000 $\mathrm{km}$-for a reason that will soon be apparent, and this means that general relativistic effects are not important here. The ones we can see optically shine because they have not yet rid themselves of all the energy of gravitational collapse.

For some time it was a real puzzle to explain why this dead star did not collapse entirely. We have seen that the quantum mechanical kinetic energy provides the requisite "internal pressure", and the person who modelled this correctly was Chandrasekhar in 
1931 [C]. There is, however, a slight twist from the neutron star model, which requires some explanation.

The main force among electrons and nuclei is electrostatic-not gravitational. But with a grain of faith we can suppose that the electric potential is cancelled locally, i.e., local neutrality is strongly enforced. This leaves only the gravitational potential, which is additive because there are no \pm signs. Ideally we would like to incorporate both forces, but we shall retain only the gravitational force. The nuclei, as before, can be considered to be almost motionless but the electrons move with high speeds (because of the high density) and therefore have to be treated relativistically.

With this information we can, with another grain of faith, suppose that the Hamiltonian $H_{N}$ in (8.2) is relevant, but with the following identifications. The number of electrons is $N$ and the mass $m$ that appears in the kinetic energy, $T_{o p}$, is the electron mass. On the other hand, the gravitational force comes mostly from the nuclei (because of their large mass), and therefore $\kappa=$ $G M^{2}$ with $M$ now being the mass in the star per electron, i.e., $M=M_{\text {nucleus }} / z_{\text {nucleus }}$, which is much bigger than the electron's mass and which is typically about twice $M_{n}$. It is the presence of the very small $m$ in the kinetic energy which make the radius bigger for the solution of $(8.5),(8.6)$; by scaling, the radius is proportional to $1 / \mathrm{m}$ for a fixed $N$. The critical $\tau$ does not change (except insofar as $M \neq M_{n}$ ).

The proper model to analyze, of course, would be one with two kinds of particles (electrons and nuclei) and with two kinds of forces (electrostatic and gravitational), but this remains an open problem. There are some remarks about this problem in [LT3]. Another thing one would like to analyze is the effect of positive temperature. For nonrelativistic particles, both kinds of modifications have been achieved, in a certain limit, by Hertel, Narnhofer and Thirring [HT, HNT, TW]. See also Messer's book [M]. The relativistic case with both kinds of forces is still an open problem.

Chandrasekhar, of course, did not analyze the Hamiltonian $H_{N}$ as we did here. He started instead with the Euler-Lagrange equation of the Thomas-Fermi approximation, (8.14) or (8.15). In fact, it was the following physical interpretation of (8.14) that motivated Chandrasekhar: If we take the gradient of both sides and then multiply by $-\rho(x)$, the right side becomes the gravitational force at a point $x$ in the star. The left side can be interpreted as the gradient of the pressure, $P$, of a "quantum mechanical fluid." This fluid has an "equation of state" (i.e., the pressure, $P$ as a function of the density, $\rho$ ) given by the following formula that is 
valid for all fluids.

$$
P(\rho)=\rho j^{\prime}(\rho)-j(\rho)=\rho^{2} \frac{\partial}{\partial \rho} \frac{j(\rho)}{\rho} .
$$

This balancing of forces, i.e., the "gravitational-hydrostatic equilibrium," was Chandrasekhar's starting point.

From this point of view there is an interesting contrast between the bosonic case (well approximated by the Hartree theory (8.18)) and the fermionic case (well approximated by the Thomas-Fermi theory (8.5)). The latter energy can be thought of as that of a simple fluid which has an "equation of state." This is how physicists think of the matter, even though it is quantum mechanics that produces the pressure. The bosonic energy (8.18) has no such interpretation. Quantum mechanics shows itself to the bitter end because the Hartree energy still has gradients in it. The concept of an "equation of state" cannot be used for high density bosonic matter in its ground state.

This brings us to the end of the stability of matter story. If one more hyperbolic remark be permitted, it can be said that

Quantum mechanics is a bizarre theory, invented to explain atoms. As far as we know today it is capable of explaining everything about ordinary matter (chemistry, biology, superconductivity), sometimes with stunning numerical accuracy. But it also says something about the occurrence of the most spectacular event in the cosmos-the supernova. The range is 57 orders of magnitude!

\section{REFERENCES}

[AI] American Institute of Physics Handbook, McGraw-Hill, New York, 1972 third ed., p. 7-6.

[AM] P. Armbruster and G. Münzenberg, Creating superheavy elements, Scientific American 260 (1989), 66-72.

[AB] J. Auchmuty and R. Beals, Variational solution of some nonlinear free boundary problems, Arch. Rat. Mech. Anal. 43 (1971), 255-271. See also Models of rotating stars, Astrophys. J. 165 (1971), L79-L82.

[B] G. Baym, Neutron stars, in Enclyclopedia of Physics, (R. G. Lerner and G. L. Trigg eds.) Addison-Wesley, London, 1981, pp. 659-660.

[BM] M. Born, Quantenmechanik der Stossvorgänge, Z. Phys. 38 (1926), 803-827.

[CH] S. Chandrasekhar, The maximum mass of ideal white dwarfs, Astrophys. J. 74 (1931), 81-82. See also On stars, their evolution and stability, Rev. Mod. Phys. 56 (1984), 137-147.

[CO] J. Conlon, The ground state energy of a classical gas, Comm. Math. Phys. 94 (1984), 439-458.

[CLY] J. G. Conlon, E. H. Lieb and H-T. Yau, The $N^{7 / 5}$ law for charged bosons, Comm. Math. Phys. 116 (1988), 417-448. 
[DA] I. Daubechies, An uncertainity principle for fermions with generalized kinetic energy, Comm. Math. Phys. 90 (1983), 511-520.

[DAL] I. Daubechies and E. H. Lieb, One electron relativistic molecules with Coulomb interactions, Comm. Math. Phys. 90 (1983), 497-510.

[D] F. J. Dyson, Ground state energy of a finite system of charged particles, J. Math. Phys. 8 (1967), 1538-1545.

[DL] F. J. Dyson and A. Lenard, Stability of matter. I and II, J. Math. Phys. 8 (1967), 423-434; ibid 9 (1968), 698-711.

[FD] C. Fefferman and R. de la Llave, Relativistic stability of matter. I., Rev. Math. Iberoamericana 2 (1986), 119-215.

[FE] E. Fermi, Un metodo statistico per la determinazione di alcune priorieta dell'atomo, Atti Acad. Naz. Lincei, Rend. 6 (1927), 602-607.

[FR] A. P. French, Atoms, in Encyclopedia of Physics, (R. G. Lerner and G. L. Trigg eds.), Addison-Wesley, London (1981), p. 64.

[H] I. Herbst, Spectral theory of the operator $\left(p^{2}+m^{2}\right)^{1 / 2}-z e^{2} / r$, Comm. Math. Phys. 53 (1977), 285-294. Errata, ibid. 55 (1977), 316.

[HNT] P. Hertel, H. Narnhofer and W. Thirring, Thermodynamic functions for fermions with gravostatic and electrostatic interactions, Comm. Math. Phys. 28 (1972), 159-176.

[HT] P. Hertel and W. Thirring, Free energy of gravitating fermions, Comm. Math. Phys. 24 (1971), 22-36.

[J] J. H. Jeans, The mathematical theory of electricity and magnetism, Cambridge Univ. Press, Cambridge, third edition, 1915, p. 168.

[JM] M. Jammer, The conceptual development of quantum mechanics, McGrawHill, New York, 1966.

[K] T. Kato, Perturbation theory for linear operators, Springer-Verlag, Heidelberg, 1966. See Remark 5.12 on p. 307.

[KS] H. Kalf, U.-W. Schminke, J. Walter and R. Wüst, On the spectral theory of Schrödinger and Dirac operators with strongly singular potentials, Lecture Notes in Math., vol. 448 Springer-Verlag, Berlin and New York, 1974, pp. 182-226.

[LE] A. Lenard, Lectures on the Coulomb stability problem, Lecture Notes in Physics 20 (1973), 114-135.

[L1] E. H. Lieb, Stability of matter, Rev. Mod. Phys. 48 (1976), 553-569.

[L2] _ On characteristic exponents in turbulence, Comm. Math. Phys. 92 (1984), 473-480.

[L3] _ Thomas-Fermi and related theories of atoms and molecules, Rev. Mod. Phys. 53 (1981), 603-641; errata ibid 54 (1982), 311.

[L4] _ Bound on the maximum negative ionization of atoms and molecules, Phys. Rev. 29A (1984), 3018-3028. A summary is in Phys. Rev. Lett. 52 (1984), 315-317.

[L5] _ The $N^{5 / 3}$ law for bosons, Phys. Lett. A 70 (1979), 71-73.

[LL] E. H. Lieb and J. L. Lebowitz, The constitution of matter: existence of thermodynamics for systems composed of electrons and nuclei, Adv. in Math. 9 (1972), 316-398.

[LO] E. H. Lieb and S. Oxford, An improved lower bound on the indirect Coulomb energy, Int. J. Quant. Chem. 19 (1981), 427-439. 
[LS] E. H. Lieb and B. Simon, The Thomas-Fermi theory of atoms, molecules and solids, Adv. in Math. 23 (1977), 22-116.

[LT1] E. H. Lieb and W. E. Thirring, Bound for the kinetic energy of fermions which proves the stability of matter, Phys. Rev. Lett. 35 (1975), 687-689. Errata ibid. 35 (1975), 1116.

[LT2] _ Inequalities for the moments of the eigenvalues of the Schrödinger Hamiltonian and their relation to Sobolev inequalities, in Studies in Mathematical Physics, (E. Lieb, B. Simon and A. Wightman, eds.), Princeton Univ. Press, Princeton, New Jersey, 1976, pp. 269-330.

[LT3] _ _ Gravitational collapse in quantum mechanics with relativistic kinetic energy, Ann. of Phys. (NY) 155 (1984), 494-512.

[LY1] E. H. Lieb and H-T. Yau, The stability and instability of relativistic matter, Comm. Math. Phys. 118 (1988), 177-213. A summary is in Many-body stability implies a bound on the fine structure constant, Phys. Rev. Lett. 61 (1988), 1695-1697.

[LY2] _ _ The Chandrasekhar theory of stellar collapse as the limit of quantum mechanics, Comm. Math. Phys. 112 (1987), 147-174. A summary is in A rigorous examination of the Chandrasekhar theory of stellar collapse, Astrophys. J. 323 (1987), 140-144.

[M] J. Messer, Temperature dependent Thomas-Fermi theory, Lectures Notes in Physics no. 147, Springer-Verlag, Berlin and New York, 1981.

[P] W. Pauli, Über den Zusammenhang des Abschlusses der Elektronengruppen im Atom mit der Komplexstruktur der Spektren, Z. Phys. 31 (1925), 765-785.

[RB] R. Ruffini and S. Bonazzola, Systems of self-gravitating particles in general relativity and the concept of equation of state, Phys. Rev. 187 (1969), 17671783.

[SE] E. Schrödinger, Quantisierung als Eigenwertproblem, Ann. Phys. 79 (1926), 361-376. See also ibid. 79 (1926), 489-527; 80 (1926), 437-490; 81 (1926), 109-139.

[ST] S. L. Shapiro and S. A. Teukolsky, Black holes, white dwarfs and neutron stars, Wiley, New York, 1983.

[T] L. H. Thomas, The calculation of atomic fields, Proc. Cambridge Philos. Soc. 23 (1927), 542-548.

[TW] W. Thirring, A course in mathematical physics, vol. 4, Springer-Verlag, Berlin and New York, 1983.

Departments of Mathematics and Physics, Princeton University, Princeton, New Jersey 08544 
